J. DIFFERENTIAL GEOMETRY

64 (2003) 359-423

\title{
FROM CONSTANT MEAN CURVATURE HYPERSURFACES TO THE GRADIENT THEORY OF PHASE TRANSITIONS
}

\author{
FRANK PACARD \& MANUEL RITORÉ
}

\begin{abstract}
Given a nondegenerate minimal hypersurface $\Sigma$ in a Riemannian manifold, we prove that, for all $\varepsilon$ small enough there exists $u_{\varepsilon}$, a critical point of the Allen-Cahn energy $E_{\varepsilon}(u)=\varepsilon^{2} \int|\nabla u|^{2}+\int\left(1-u^{2}\right)^{2}$, whose nodal set converges to $\Sigma$ as $\varepsilon$ tends to 0 . Moreover, if $\Sigma$ is a volume nondegenerate constant mean curvature hypersurface, then the same conclusion holds with the function $u_{\varepsilon}$ being a critical point of $E_{\varepsilon}$ under some volume constraint.
\end{abstract}

\section{Introduction}

Let $\Omega \subset \mathbb{R}^{n+1}, n \geqslant 1$, be an open bounded set with smooth boundary $\partial \Omega$. For any $\varepsilon>0$ and any function $u: \Omega \rightarrow \mathbb{R}$ such that $u \in H^{1}(\Omega)$, we consider the energy

$$
E_{\varepsilon}(u):=\varepsilon^{2} \int_{\Omega}|\nabla u|^{2} d x+\int_{\Omega}\left(1-u^{2}\right)^{2} d x
$$

being understood that $E_{\varepsilon}(u)=\infty$ if $u \notin L^{4}(\Omega)$. We also consider the constraint

$$
V(u):=\int_{\Omega} u d x
$$

Given $c_{0} \in(-1,1)$, we are interested in the critical points of $E_{\varepsilon}$ subject to the constraint $V(u)=c_{0}|\Omega|$, where $|\Omega|$ denotes the volume

Received 11/28/2002. 
of $\Omega$. Any critical point of this variational problem is a solution to

$$
\begin{cases}\varepsilon^{2} \Delta u+2\left(u-u^{3}\right)=\varepsilon \lambda, & \text { in } \Omega \\ \partial_{\nu} u=0, & \text { on } \partial \Omega,\end{cases}
$$

where $\nu$ denotes a unit vector field normal to $\partial \Omega$ and where $\varepsilon \lambda \epsilon$ $\mathbb{R}$ corresponds to the Lagrange multiplier associated to the constraint $V(u)=c_{0}|\Omega|$. One can also ignore the volume constraint, in which case a critical point would satisfy Equation (1.1) with $\lambda=0$.

Since classical methods of the calculus of variation apply, there is no difficulty in finding minimizers of $E_{\varepsilon}$. The real issue is the study of the asymptotic behavior of the minimizers (or more generally of the critical points) of $E_{\varepsilon}$ as the parameter $\varepsilon$ tends to 0 . There has been a number of important work on this question over the last two decades and the basic result can be described as follows: Assume that $\left(\varepsilon_{k}\right)_{k \geqslant 0}$ tends to 0 and let $\left(u_{k}\right)_{k \geqslant 0}$ be a sequence of minimizers of $E_{\varepsilon_{k}}$ under the constraint $V(u)=c_{0}|\Omega|$. Then, up to a subsequence, one can assume that $\left(\left|u_{k}\right|\right)_{k \geqslant 0}$ converges a.e. to the constant function 1 . In the definition of the energy $E_{\varepsilon}$, the role of the term

$$
\int_{\Omega}\left(1-u^{2}\right)^{2} d x
$$

is precisely to force the sequence of functions $\left(\left|u_{k}\right|\right)_{k \geqslant 0}$ to converge to 1 when the parameter $\varepsilon_{k}$ tends to 0 . Extracting subsequences if this is necessary, we can define $\Omega^{+}$(resp. $\Omega^{-}$) to be the set of points where $u_{k}$ converges to +1 (resp. -1 ). The subsets $\Omega^{ \pm}$are not arbitrary since the constraint $V\left(u_{k}\right)=c_{0}|\Omega|$ forces $\Omega^{ \pm} \subset \Omega$ to satisfy

$$
\left|\Omega^{+}\right|-\left|\Omega^{-}\right|=c_{0}|\Omega| \text {. }
$$

Now, the role of the Dirichlet integral

$$
\varepsilon^{2} \int_{\Omega}|\nabla u|^{2} d x
$$

in the definition of $E_{\varepsilon}$ forces the interface between the subsets $\Omega^{+}$and $\Omega^{-}$to be "as small as possible", since this is where the gradient of the function $u_{k}$ will concentrate when $\varepsilon_{k}$ tends to 0 . More precisely

$$
N:=\partial \Omega^{+} \cap \Omega=\partial \Omega^{-} \cap \Omega
$$


can be shown to be a minimizer of the isoperimetric problem: Minimize amongst all domains $D \subset \Omega$ the $n$-dimensional Hausdorff measure $\mathcal{H}^{n}(\partial D)$ of the boundary $\partial D$ subject to the volume constraint

$$
|D|=\frac{1+c_{0}}{2}|\Omega|
$$

We refer to [16], [15], [12], [3], [4], [21] for more precise statements. From a purely analytic point of view, $N$ can be understood as the limit of the nodal sets of the functions $u_{k}$, as $k$ tends to $+\infty$.

\section{Statement of the problem}

It is interesting to generalize the above problem first by considering instead of $\Omega \subset \mathbb{R}^{n+1}$, any compact Riemannian manifold with or without smooth boundary and also by replacing the nonlinearity $\left(1-u^{2}\right)^{2}$ by a more general one.

Hence, in this paper, we consider $(M, g)$ to be a $(n+1)$-dimensional compact Riemannian manifold with or without smooth boundary. In the case where $\partial M$, the boundary of $M$, is not empty, we can assume without loss of generality that $M$ is a subdomain of a larger Riemannian manifold $(\widetilde{M}, \widetilde{g})$, with $\widetilde{g}_{\mid M}=g$. In particular, $\partial M$ is a smooth hypersurface of $\widetilde{M}$.

Let $W: \mathbb{R} \rightarrow \mathbb{R}$ be a smooth function which is positive away from $u= \pm 1$. We assume that

$$
W( \pm 1)=0,
$$

so that the infimum of $W$ is achieved at the points $u= \pm 1$. Further assume that these points are nondegenerate critical points of $W$. In other words

$$
W^{\prime \prime}( \pm 1)>0
$$

For any $\varepsilon>0$ and any function $u: M \longrightarrow \mathbb{R}$, such that $u \in H^{1}(M)$, we define the energy

$$
E_{\varepsilon}(u):=\varepsilon^{2} \int_{M}|\nabla u|_{g}^{2} d v_{g}+\int_{M} W(u) d v_{g},
$$

where $\nabla$ denotes the gradient and $d v_{g}$ the volume form on $M$ associated to the Riemannian metric $g$. As usual, we agree that $E_{\varepsilon}(u)=\infty$ when 
$W(u) \notin L^{1}(M)$. We also define the volume constraint

$$
V(u):=\int_{M} u d v_{g}
$$

Granted the above definitions, there are two closely related variational problems we can consider:

1. We can consider the critical points of the energy $u \longrightarrow E_{\varepsilon}(u)$, which are solutions of

$$
-\varepsilon^{2} \Delta_{g} u+\frac{1}{2} W^{\prime}(u)=0,
$$

in $M$, where $\Delta_{g}$ is the Laplace-Beltrami operator in $M$. Moreover, if $\partial M \neq \emptyset$ then the additional condition

$$
\partial_{\nu_{\partial M}} u=0
$$

must hold on $\partial M$, where $\nu_{\partial M}$ denotes the unit vector field normal to $\partial M$. This problem is related to the Allen-Cahn equation [2] and it is well-known that, as $\varepsilon$ tends to 0 , the interfaces (i.e., the nodal sets of the solutions of (2.5)) converge to minimal hypersurfaces. Concerning this variational problem, the question we would like to address in this paper is the following:

Assume that $N \subset M$ is a minimal hypersurface. Does $N$ appear as the limit, as the parameter $\varepsilon$ tends to 0 , of the nodal sets of a sequence of critical points of $E_{\varepsilon}$ ?

2. Given $c_{0} \in(-1,1)$, we can consider the critical points of the energy $u \longrightarrow E_{\varepsilon}(u)$ under the constraint $V(u)=c_{0}|M|$, where $|M|$ denotes the volume of $M$. This time, such a critical point $u$ is solution of

$$
-\varepsilon^{2} \Delta_{g} u+\frac{1}{2} W^{\prime}(u)=\varepsilon \lambda,
$$

in $M$, where $\varepsilon \lambda \in \mathbb{R}$ corresponds to the Lagrange multiplier associated to the constraint $V(u)=c_{0}|M|$. Moreover, $u$ satisfies (2.6) on $\partial M$ if $\partial M \neq \emptyset$. According to [19], [8], the energy $E_{\varepsilon}$ corresponds to the total energy of a fluid within the Wan der Waals-Cahn-Hilliard theory of phase transitions. The Lagrange 
multiplier $\varepsilon \lambda$, which appears in (2.7), is known in the physics literature as the chemical potential of the density configuration $u$. Now, the question we would like to address becomes:

$$
\begin{aligned}
& \text { Assume that } N \subset M \text { is a constant mean curvature } \\
& \text { hypersurface. Does } N \text { appear as the limit, as the } \\
& \text { parameter } \varepsilon \text { tends to } 0 \text {, of the nodal sets of a se- } \\
& \text { quence of critical points of } E_{\varepsilon} \text { subject to the constraint } \\
& V(u)=c_{0}|M| \text { ? }
\end{aligned}
$$

Before we proceed, let us observe that, in both problems, we are not only looking for minimizers of $E_{\varepsilon}$ but more generally for critical points.

Remark 2.1. If the infimum of the function $W$ is achieved at exactly two points $u_{ \pm}$, there is no loss of generality in considering that $u_{ \pm}= \pm 1$ since we can always reduce to this case by considering $u \mapsto W(a u+b)$ where $a$ and $b$ are chosen appropriately.

\section{Definitions and Preliminaries}

\subsection{Admissible hypersurfaces in $M$}

Obviously if $N$ is the nodal set of some function $u$ which is defined in $M$ and if 0 is a regular value of $u$ then $M-N$ is the union of

$$
M^{+}(N):=u^{-1}((0,+\infty)) \quad \text { and } \quad M^{-}(N):=u^{-1}((-\infty, 0)) .
$$

We shall associate to $N$ the unit normal vector field which points into $M^{+}(N)$. In the case where $M$ has a boundary, it may happen that $N$ also has a boundary $\partial N \subset \partial M$. In this case, if $N$ is the nodal set of the function $u$ and if in addition the function $u$ has 0 Neumann boundary condition on $\partial M$, then for all $p \in \partial N \subset \partial M$, the normal vector to $N$ at $p$ and the normal vector to $\partial M$ at $p$ are orthogonal. This later condition is standard in the study of minimal and constant mean curvature hypersurfaces. Indeed, it is well-known that smooth hypersurfaces $N$ which are stationary points of the area functional (possibly with a volume constraint) and have a boundary $\partial N \subset \partial M$, satisfy the later orthogonality condition. This motivates the following:

Definition 3.1. A smooth embedded hypersurface $N \subset M$ (not necessarily connected) is admissible if $N$ is the nodal set of a smooth 
function $u$ for which 0 is a regular value of $u$ and which, in the case where $M$ has a boundary, has 0 Neumann boundary condition.

A hypersurface $N \subset M$ which separates $M$ into two regions $M^{ \pm}(N)$, and which meets $\partial M$ orthogonally in the case where $N$ has a nonempty boundary, is easily shown to be admissible by using partitions of unity. Observe that $N$ may have many connected components.

\subsection{The Jacobi operator}

Before we introduce our next definition, we recall a few basic facts about the study of constant mean curvature hypersurfaces $N$ in a Riemannian manifold $(M, g)$. To begin with, let us recall that the Jacobi operator, that is the linearized mean curvature operator about $N$, is given by

$$
\mathcal{L}_{N}:=\Delta_{N}+\left|A_{N}\right|^{2}+\operatorname{Ric}_{g}\left(\nu_{N}, \nu_{N}\right),
$$

where $\Delta_{N}$ is the Laplace-Beltrami operator on $N,\left|A_{N}\right|^{2}$ denotes the norm of the second fundamental form of $N$, $\mathrm{Ric}_{g}$ is the Ricci tensor of $M$ and $\nu_{N}$ is a unit normal to $N$.

Given any (smooth) small function $w$ on $N$, we can consider the hypersurface $N(w)$ which is the normal graph on $N$ of the function $w$ (the image of $N$ by the map $p \in N \mapsto \exp _{p}\left(w(p) \nu_{N}(p)\right)$ ). If $H(w)$ denotes the mean curvature of $N(w)$, defined as the arithmetic mean of the principal curvatures, then the linear operator $\mathcal{L}_{N}$ is the differential of $w \mapsto n H(w)$ at $w \equiv 0$.

When $\partial N$ is empty, solutions of the homogeneous problem

$$
\mathcal{L}_{N} w=0
$$

on $N$ are called Jacobi fields. When $\partial N$ is not empty, we further assume that $N$ meets $\partial M$ orthogonally, then Jacobi fields are the solutions of $\mathcal{L}_{N} w=0$ in $N$ which satisfy the boundary condition

$$
\mathcal{B}_{N} w:=\partial_{\nu_{\partial M}} w+A_{\partial M}\left(\nu_{N}, \nu_{N}\right) w=0,
$$

on $\partial N$, where $A_{\partial M}$ is the second fundamental form of $\partial M$ in $\widetilde{M}$. Equation (3.4) has its origin in the requirement that all the hypersurfaces we are looking at meet $\partial M$ orthogonally and this should be true for the hypersurfaces generated by the flow associated to a vector field $X$ satisfying $X=w \nu_{N}$ on $N$.

Minimal hypersurfaces are critical points of the area functional while constant mean curvature hypersurfaces are critical points of the area 
functional with respect to deformations that keep constant the volume enclosed by the hypersurface. Consider a deformation of the hypersurface $N$ by the flow generated by a vector field $X$. The second variation formula for the area functional is then given by

$$
X \longrightarrow-\int_{N} w \mathcal{L}_{N} w d a_{g}+\int_{\partial N} w \mathcal{B}_{N} w d s_{g}
$$

where the function $w:=g\left(\nu_{N}, X\right)$. If one considers a deformation which is volume preserving up to first order, then the function $w$ also has to satisfy

$$
\int_{N} w d a_{g}=0
$$

We refer to [5] or [18] for a derivation of the second variation of the area functional in a Riemannian manifold. Here $d a_{g}$ and $d s_{g}$ are the volume forms on $N$ and $\partial N$ which are induced by the metric $g$.

\subsection{Nondegeneracy}

The previous definitions being understood, we can now give the notions of nondegeneracy which are associated to the two problems we are interested in. To begin with let us define the notion of nondegenerate minimal hypersurface:

Definition 3.2. An admissible minimal hypersurface $N$ is said to be nondegenerate if there are no nontrivial solutions $w \in \mathcal{C}^{2, \alpha}(N)$ of

$$
\mathcal{L}_{N} w=0,
$$

in $N$, with $\mathcal{B}_{N} w=0$ on $\partial N$ if $N$ has a boundary.

The notion of nondegeneracy for minimal hypersurfaces is standard. Consider the Jacobi operator

$$
\mathcal{L}_{N}:\left[\mathcal{C}^{2, \alpha}(N)\right]_{0} \longrightarrow \mathcal{C}^{0, \alpha}(N),
$$

where the subscript 0 is meant to point out that functions in $\left[\mathcal{C}^{2, \alpha}(N)\right]_{0}$ satisfy $\mathcal{B}_{N} w=0$ on $\partial N$ when this latter is not empty. Nondegeneracy is equivalent to the fact that the operator $\mathcal{L}_{N}$ is injective. This operator being self-adjoint and elliptic, nondegeneracy is also equivalent to the invertibility of the operator $\mathcal{L}_{N}$ defined in (3.5). On a more geometric point of view, if $N$ is a nondegenerate minimal hypersurface, the implicit 
function theorem ensures that it is possible to find a hypersurface $\widetilde{N}$ which is close to $N$ and whose mean curvature $\widetilde{H}$ is prescribed, close to the mean curvature $H$ of $N$.

We will also need the notion of volume-nondegenerate constant mean curvature hypersurface:

Definition 3.3. An admissible constant mean curvature hypersurface $N$ is said to be volume-nondegenerate if there are no nontrivial solutions $(w, c) \in \mathcal{C}^{2, \alpha}(N) \times \mathbb{R}$ of

$$
\mathcal{L}_{N} w+c=0, \quad \text { and } \quad \int_{N} w d v_{g}=0,
$$

in $N$, with $\mathcal{B}_{N} w=0$ on $\partial N$ if $N$ has a boundary.

The notion of volume-nondegeneracy is less standard and perhaps requires some explanation. This time, we consider the extended-operator

$$
\begin{aligned}
L_{N}:\left[\mathcal{C}^{2, \alpha}(N)\right]_{0} \times \mathbb{R} & \longrightarrow \mathcal{C}^{0, \alpha}(N) \times \mathbb{R} \\
(w, c) & \longmapsto\left(\mathcal{L}_{N} w+c, \int_{N} w d a_{g}\right) .
\end{aligned}
$$

Thus, volume-nondegeneracy is equivalent to the fact that the operator $L_{N}$ is injective. Observe that $L_{N}$ is self-adjoint with respect to the scalar product

$$
\langle(v, c),(w, d)\rangle:=\int_{N} v w d a_{g}+c d,
$$

in $L^{2}(N) \times \mathbb{R}$. The operator $L_{N}$ being clearly elliptic, volume-nondegeneracy is also equivalent to the invertibility of the operator $L_{N}$ defined in (3.6). From a geometric point of view, if $N$ is a constant mean curvature volume-nondegenerate hypersurface, the implicit function theorem ensures that it is possible to find a hypersurface $\widetilde{N}$ which is close to $N$, whose mean curvature $\widetilde{H}$ is, up to a constant function, prescribed close to $H$ the mean curvature of $N$ and such that the volume enclosed by this hypersurface $M^{+}(\widetilde{N})$ is prescribed close to $M^{+}(N)$, the volume enclosed by $N$. Hence, it is possible to prescribe the volume enclosed by $\widetilde{N}$ and, up to a constant function, the mean curvature of $\widetilde{N}$.

\section{Statement of the result}

The previous definitions being understood, we can now state the results we have obtained concerning both $(\mathrm{P}-1)$ and $(\mathrm{P}-2)$. 
We have the:

Theorem 4.1. Assume that $N \subset M$ is an admissible nondegenerate minimal hypersurface. Then, there exists $\varepsilon_{0}>0$ and for all $\varepsilon \in\left(0, \varepsilon_{0}\right)$ there exists $u_{\varepsilon}$, critical point of $u \longrightarrow E_{\varepsilon}(u)$, such that $u_{\varepsilon}$ converges uniformly to 1 on compact subsets of $M^{+}(N)$ (resp. to -1 on compact subsets of $\left.M^{-}(N)\right)$.

Let us mention the work of M. Kowalczyk [11] where a similar result is obtained when $M$ is a two dimensional domain of $\mathbb{R}^{2}$ and $N$ is a line segment.

We will also prove the:

Theorem 4.2. Assume that $N \subset M$ is an admissible volumenondegenerate constant mean curvature hypersurface. Then, there exists $\varepsilon_{0}>0$ and for all $\varepsilon \in\left(0, \varepsilon_{0}\right)$ there exists $u_{\varepsilon}$, critical point of $u \longrightarrow E_{\varepsilon}(u)$ under the constraint $V(u)=\left|M^{+}(N)\right|-\left|M^{-}(N)\right|$, such that $u_{\varepsilon}$ converges uniformly to 1 on compact subsets of $M^{+}(N)$ (resp. to -1 on compact subsets of $\left.M^{-}(N)\right)$.

It is in general extremely hard to check whether a given minimal hypersurface (resp. constant mean curvature hypersurface) is nondegenerate (resp. volume-nondegenerate). Hopefully, first observe that both nondegeneracy and volume-nondegeneracy are "open conditions", namely are stable under small perturbation of the metric. Moreover, in [23], B. White has proved that minimal hypersurfaces are nondegenerate for a generic choice of the metric. It follows from similar arguments that volume-nondegeneracy also holds for a generic choice of the metric.

The solutions constructed in Theorem 4.2 are solutions of

$$
-\varepsilon^{2} \Delta u_{\varepsilon}+\frac{1}{2} W^{\prime}\left(u_{\varepsilon}\right)=\varepsilon \lambda_{\varepsilon}
$$

As a byproduct of our construction, we obtain a precise expansion of $u_{\varepsilon}$ in terms of $\varepsilon$. We also get the expansion of the Lagrange multiplier $\lambda_{\varepsilon}$

$$
\lambda_{\varepsilon}=\frac{1}{2} c_{\star} n H_{N}+\mathcal{O}(\varepsilon),
$$

where $H_{N}$ is the mean curvature of the limit interface $N$ and where the constant $c_{\star}$ is given by

$$
c_{\star}:=\int_{-1}^{+1} \sqrt{W(s)} d s .
$$


Finally, in both problems, the expansion of the energy $E_{\varepsilon}\left(u_{\varepsilon}\right)$ of the solutions we construct, is given by

$$
E_{\varepsilon}\left(u_{\varepsilon}\right)=2 \varepsilon c_{\star}|N|+\mathcal{O}\left(\varepsilon^{2}\right) .
$$

where $|N|$ is the volume of the interface $N$. These expansions agree with the expansions which have already been obtained in [13] in the case where $u_{\varepsilon}$ are minimizers of $E_{\varepsilon}$ subject to the constraint $V=\left|M^{+}(N)\right|-$ $\left|M^{-}(N)\right|$.

Unfortunately, in many interesting cases and despite the genericity of these notions, minimal hypersurfaces are degenerate and constant mean curvature hypersurfaces are volume-degenerate. This is for example the case when there is a nontrivial group of isometries acting on $M$. It is well-known that any $\left(\phi_{t}\right)_{t \in(-1,1)}$ smooth one-parameter group of isometries of $M\left(\phi_{s} \circ \phi_{t}=\phi_{s+t}\right)$ gives rise to a Jacobi field on $N$ (when $M$ has a nonempty boundary, we ask that these isometries preserve globally $\partial M)$. Actually, the Jacobi field $w$ is explicitly given by $w:=g\left(\nu_{N}, X\right)$, where $\nu_{N}$ is the normal vector field to $N$ and where $X:=\partial_{t} \phi_{t \mid t=0}$ is the Killing field corresponding to the one-parameter group of isometries $\left\{\phi_{t}\right\}_{t \in(-1,1)}$. Observe that the isometries $\phi_{t}$ preserve the volume of the regions $M^{ \pm}(N)$. Therefore, it follows from the first variation of volume that the Jacobi field $w$ has mean zero on $N$. In particular, $w$ is a nontrivial solution of $\mathcal{L}_{N} w=0$ (resp. $(w, 0)$ is a nontrivial solution of $\left.L_{N}(w, 0)=0\right)$ and the hypersurface $N$ is degenerate (resp. volumedegenerate).

In some cases, it is possible to reduce to a nondegenerate (or volumenondegenerate) problem by working in the space of functions and hypersurfaces which are equivariant with respect to the action of some finite group of symmetries. If this can be done, then the above theorems apply mutatis mutandis. We give here a short list of examples.

1. Consider $M=S^{n+1}$ the unit $(n+1)$-dimensional sphere with the standard metric and $N=S^{n}(r)$ the meridian at height $\sqrt{1-r^{2}}$. The hypersurface $N$ has constant mean curvature and is volumedegenerate since there are nontrivial Jacobi fields $w_{i}(x)=x \cdot e_{i}$, for $i=1, \ldots, n$ coming from the action of the orthogonal group. Here $e_{1}, \ldots, e_{n+1}$ is an orthonormal basis of $\mathbb{R}^{n+1}$. However, one may work with hypersurfaces and functions which are invariant under the action of the $n$ hyperplanar symmetries

$$
I_{i}:\left(x_{1}, \ldots, x_{i}, \ldots, x_{n+1}\right) \longrightarrow\left(x_{1}, \ldots,-x_{i}, \ldots, x_{n+1}\right)
$$


for $i=1, \ldots, n$. Namely, hypersurfaces $\widetilde{N} \subset S^{n+1}$ such that $I_{i}(\tilde{N})=\widetilde{N}$ and functions $u: S^{n+1} \longrightarrow \mathbb{R}$ such that $u \circ I_{i}=u$. Since none of the Jacobi fields is invariant under all the symmetries $I_{i}$, our construction applies and the conclusion of Theorem 4.2 is still valid. Moreover, when $r=1$, the equator is a minimal hypersurface and Theorem 4.1 is also valid.

2. Consider $M=B^{n+1}$ the unit ball of $\mathbb{R}^{n+1}$ endowed with the induced metric and $N$ is a spherical cap. This example can be dealt like the previous one and the result of Theorem 4.2 holds. Moreover, when $N$ is the horizontal hyperplane, Theorem 4.1 also holds.

3. Consider a flat torus $T^{n+1}$ and let $N \subset T^{n+1}$ be a pair of parallel hyperplanes. For the sake of simplicity, assume that $T^{n+1}=$ $\mathbb{R}^{n+1} / \mathbb{Z}^{n+1}$ and is identified with $\left[-\frac{1}{2}, \frac{1}{2}\right]^{n+1}$. Finally, assume that $N$ is given by $x_{n+1}= \pm \alpha$ for some fixed $\alpha \in(0,1 / 2)$. The action of vertical translations induce a nontrivial Jacobi field. Again, one may work with hypersurfaces and functions which are invariant under the action of the $n+1$ hyperplanar symmetry

$$
I_{n+1}:\left(x_{1}, \ldots, x_{i}, \ldots, x_{n+1}\right) \longrightarrow\left(x_{1}, \ldots, x_{n},-x_{n+1}\right),
$$

and reduce to a volume-nondegenerate problem to show that the conclusion of Theorem 4.1 and Theorem 4.2 are valid.

\section{Comments}

We state here a number of comments, open problems and directions for further investigations:

1. In [14], A. Malchiodi and M. Montenegro have constructed solutions of

$$
\varepsilon^{2} \Delta u-u+u^{p}=0
$$

which are defined on a 2 dimensional domain and which have 0 Neumann boundary condition. These solutions have the property that they concentrate along the boundary of the domain and they can be obtained for $\varepsilon$ belonging to some sequence of intervals which converge to 0 . Behind this result, lies a very interesting bifurcation 
phenomena which somehow prevents the construction to work for all $\varepsilon$ close enough to 0 . The analysis of A. Malchiodi and M. Montenegro relies on the precise estimate of the least eigenvalue of the linearized operator about an approximate solution, and this forces them to work in 2 dimensional domains. It would be very interesting to construct solutions of (5.1) which concentrate along a geodesic of a two dimensional manifold and also to extend their result to higher dimensional domains.

2. As already mentionned, M. Kowalczyk [11] has obtained a similar result when $M$ is a two dimensional domain of $\mathbb{R}^{2}$ and $N$ is a line segment. Our result can be also be compared to the result of C.H. Taubes [22] where solutions of the Seiberg-Witten equation concentrating along holomorphic curves are constructed, though our analysis is completely different. Very closely related to our result, is the one of S. Brendle [7] on the construction of solutions of the Ginzburg-Landau equation which concentrate along codimension 2 minimal submanifolds. In all these results, solutions to nonlinear partial differential equation which concentrate along smooth submanifolds are constructed. The concentration set is always a minimal submanifold, which has to be assumed to be nondegenerate. Hence the construction holds for a generic choice of the background metric.

3. Let us also mention the work of S. Brendle concerning Yang-Mills connections in higher dimensions [6]. However, in this result, the construction does not hold for a generic choice of the metric but rather for a fairly restricted set of metrics.

4. We have not studied the case where the hypersurfaces are singular. For example, it is known that stable minimal cones do exist in dimension $n+1 \geqslant 8$ and it would be very interesting to develop the corresponding analysis in this case.

5. We have not studied the case where there is a nontrivial group of isometries acting on $M$ and where the problem cannot be reduced to a nondegenerate problem by working in the space of functions and hypersurfaces which are equivariant with respect to some finite group of symmetries. For example, one may consider the case where $M$ is the $(n+1)$-sphere with the standard metric and $N$ is the equator. Now, let us perturb slightly the metric on $S^{n+1}$ in 
some neighborhood of the north pole. Unless the perturbed metric is invariant under the action of $I_{i}$, for $i=1, \ldots, n$ which are defined in Example (1), our method does not apply in this setting.

\section{The canonical profile}

In this section, we consider the case where $M=\mathbb{R}$ and $\lambda=0$. In this case, Equation (2.7) reduces to the following second order ordinary differential equation

$$
\partial_{t}^{2} u-\frac{1}{2} W^{\prime}(u)=0
$$

Observe that

$$
H\left(u, \partial_{t} u\right):=\left(\partial_{t} u\right)^{2}-W(u),
$$

is constant along solutions of (6.1). Using this property it is easy to check that there exists a solution of (6.1), which will be denoted by $u_{\star}$ in the remaining of the paper, and which satisfies

$$
\lim _{t \rightarrow \pm \infty} u_{\star}(t)= \pm 1, \quad \text { and } \quad u_{\star}(0)=0 .
$$

This solution corresponds to $H\left(u, \partial_{t} u\right) \equiv 0$ and is implicitly defined by

$$
t=\int_{0}^{u_{\star}(t)} \frac{d x}{\sqrt{W(x)}} .
$$

We define the indicial roots $\gamma_{ \pm}>0$ by

$$
\gamma_{ \pm}^{2}:=\frac{1}{2} W^{\prime \prime}( \pm 1)
$$

(observe that \pm 1 are minimizers of $W$ and are assumed to be nondegenerate, hence $W^{\prime \prime}( \pm 1)>0$ and this implies that $\gamma_{ \pm}$are well-defined). The asymptotics of the function $u_{\star}$ as $t$ tends to $\pm \infty$ are easy to derive by linearizing (6.1) about $u \equiv \pm 1$. We find that, for all $k \in \mathbb{N}$, there exists $c_{k}>0$ such that

$$
\left|\partial_{t}^{k}\left(u_{\star}(t)+1\right)\right| \leqslant c_{k} e^{\gamma_{-} t} \quad \text { for all } \quad t \leqslant 0,
$$

and

$$
\left|\partial_{t}^{k}\left(u_{\star}(t)-1\right)\right| \leqslant c_{k} e^{-\gamma_{+} t} \quad \text { for all } \quad t \geqslant 0 \text {. }
$$




\section{Injectivity results}

We prove some injectivity results for ordinary differential operators and partial differential operators whose potential is defined using the function $u_{\star}$.

\subsection{Preliminary results}

For all $\zeta \in \mathbb{R}$, we set

$$
L_{\zeta}:=-\partial_{t}^{2}+\zeta+\frac{1}{2} W^{\prime \prime}\left(u_{\star}\right)
$$

All the injectivity results rely on the fact that, when $\zeta=0$, the function

$$
w_{\star}:=\partial_{t} u_{\star},
$$

is a bounded positive solution of the homogeneous problem

$$
L_{0} w_{\star}=0
$$

Furthermore $w_{\star}$ decays exponentially at both $+\infty$ and $-\infty$. We introduce, for $\zeta \geqslant 0$, the indicial roots of the operator $L_{\zeta}$ at $\pm \infty$ by

$$
\gamma_{ \pm}(\zeta):=\sqrt{\zeta+\gamma_{ \pm}^{2}}
$$

where $\gamma_{ \pm}$have been defined in (6.2). These indicial roots are related to the asymptotic expansion near $\pm \infty$ of the solutions of the homogeneous problem $L_{\zeta} w=0$. For example, it follows from Cauchy's existence result for solutions of ordinary differential equations that there exist $\underline{w}$ and $\bar{w}$ solutions of $L_{\zeta} w=0$, which satisfy

$$
\lim _{t \rightarrow+\infty} e^{-\gamma_{+}(\zeta) t} \bar{w}(t)=1, \quad \text { and } \quad \lim _{t \rightarrow+\infty} e^{\gamma_{+}(\zeta) t} \underline{w}(t)=1 .
$$

Our first injectivity result reads:

Lemma 7.1. Assume that $\zeta \geqslant 0$ and let $w$ be a solution of $L_{\zeta} w=0$ which is defined on $\left(t_{1}, t_{2}\right)$. Further assume that $w\left(t_{i}\right)=0$, for $i=1,2$. Then $w \equiv 0$.

Proof. We argue by contradiction. Given $\eta \in \mathbb{R}$, observe that

$$
L_{\zeta}\left(w_{\star}+\eta w\right)=\zeta w_{\star}
$$


Now, since we have assumed that $w$ is not identically equal to 0 , one can choose $\eta \in \mathbb{R}$ such that the infimum of the function $W:=w_{\star}+\eta w$, over $\left[t_{1}, t_{2}\right]$ is equal to 0 . Observe that, since $w\left(t_{1}\right)=w\left(t_{2}\right)=0$, the infimum of $W$ is achieved at some point $t_{0} \in\left(t_{1}, t_{2}\right)$ and $L_{\zeta} W \leqslant 0$ at this point. When $\zeta>0$, this last inequality clearly contradicts (7.2). When $\zeta=0$, observe that $W$ is a solution of some second order linear ordinary differential equation and that $W\left(t_{0}\right)=\partial_{t} W\left(t_{0}\right)=0$, hence $W \equiv 0$. This contradicts the fact that $W\left(t_{i}\right) \neq 0$ for $i=1,2$. $\quad$ q.e.d.

Our second injectivity result classifies the set of $\zeta$ for which there exists a bounded solution of $L_{\zeta} w=0$, which is defined on $\mathbb{R}$ or on a half line. Given $\delta_{ \pm} \in \mathbb{R}$, we define $\delta:=\left(\delta_{-}, \delta_{+}\right)$and the function

$$
\varphi_{\delta}(t):=\left(1+e^{t}\right)^{\delta_{+}}\left(1+e^{-t}\right)^{\delta_{-}} .
$$

In particular, $\varphi_{\delta}(t) \sim e^{\delta_{+} t}$ at $+\infty$ and $\varphi_{\delta}(t) \sim e^{-\delta_{-} t}$ at $-\infty$. This definition being understood, we now prove the:

Lemma 7.2. Assume that $\zeta>0$. Let $w$ be a solution of $L_{\zeta} w=0$ which is defined on $\mathbb{R}$ (or on $\left(-\infty, t_{0}\right)$ or $\left(t_{0},+\infty\right)$, in which case we ask that $\left.w\left(t_{0}\right)=0\right)$. Further assume that $|w|$ is bounded by a constant times the function $\varphi_{\delta}$ for $\delta_{ \pm}<\gamma_{ \pm}(\zeta)$. Then $w \equiv 0$.

Proof. The proof of this result is almost identical to the proof of the previous one. The key observation is that, under the above assumptions, any solution of $L_{\zeta} w=0$ defined on a half line decays faster than $w_{\star}$ at infinity. This follows at once from the fact that the indicial roots $\gamma_{ \pm}(\zeta)$ of $L_{\zeta}$ are larger than the indicial roots $\gamma_{ \pm}$of $L_{0}$.

For example, assume that $w$ is defined on $\left(t_{0},+\infty\right)$. Any solution of the homogeneous problem $L_{\zeta} w=0$ is a linear combination of $\bar{w}$, a solution which blows up exponentially at $+\infty$ like $e^{\gamma_{+}(\zeta) t}$, and $\underline{w}$, the solution which decays exponentially at $+\infty$ like $e^{-\gamma_{+}(\zeta) t}$. Since $|w|$ is bounded by $e^{\delta_{+} t}$ for some $\delta_{+}<\gamma_{+}(\zeta)$, we conclude that $w$ is collinear to $\underline{w}$. Now, $\gamma_{+}(\zeta)>\gamma_{+}$hence $w$ decays faster than $w_{\star}$ at $+\infty$. Once this is known, the proof of the result reduces to the proof of Lemma 7.1.

q.e.d.

The set of solutions of $L_{\zeta} w=0$ is two dimensional and there exists a unique $w_{\zeta}^{-}$solution of $L_{\zeta} w_{\zeta}^{-}=0$ which is defined on all $\mathbb{R}$ and which satisfies

$$
\lim _{t \rightarrow-\infty} e^{-\gamma_{-}(\zeta) t} w_{\zeta}^{-}(t)=1
$$


As already mentioned, this essentially follows from Cauchy's existence result for solutions of ordinary differential equations. When $\zeta>0$, the previous Lemma implies that the function $w_{\zeta}^{-}$does not vanish and furthermore blows up exponentially at $+\infty$ like $t \rightarrow e^{\gamma_{+}(\zeta) t}$. Similarly, there exists a unique $w_{\zeta}^{+}$solution of $L_{\zeta} w_{\zeta}^{+}=0$ on $\mathbb{R}$ which satisfies

$$
\lim _{t \rightarrow+\infty} e^{\gamma_{+}(\zeta) t} w_{\zeta}^{+}(t)=1
$$

Again, when $\zeta>0$, this function does not vanish and blows up exponentially at $-\infty$ like $t \rightarrow e^{-\gamma_{-}(\zeta) t}$.

\subsection{Injectivity results}

Assume that $(N, h)$ is a compact $n$-dimensional Riemannian manifold with or without boundary. Further assume that $(N, h)$ is at least $\mathcal{C}^{1, \alpha}$. This means that one can choose local coordinate charts on $N$ in which the coefficients of the metric $h$ are $\mathcal{C}^{1, \alpha}$ functions. We define on the product space $\mathbb{R} \times N$ the partial differential operator

$$
\mathfrak{L}_{h}:=-\partial_{t}^{2}-\Delta_{h}+\frac{1}{2} W^{\prime \prime}\left(u_{\star}\right)
$$

where $\Delta_{h}$ is the Laplace-Beltrami operator on $(N, h)$. Using the result of Lemma 7.1, we get the:

Corollary 7.3. Assume that $w$ is a solution of $\mathfrak{L}_{h} w=0$ which is defined on $\left(t_{1}, t_{2}\right) \times N$. Further assume that $w=0$ on $\left\{t_{i}\right\} \times N$, for $i=1,2$ and that $w$ has 0 Neumann boundary data on $\left(t_{1}, t_{2}\right) \times \partial N$ if $\partial N$ is not empty. Then $w \equiv 0$.

Proof. We denote by $\left(\phi_{j}, \lambda_{j}\right)_{j \geqslant 0}$ the eigendata of $\Delta_{h}$ (with Neumann boundary conditions when the boundary of $N$ is not empty). Namely

$$
\Delta_{h} \phi_{j}=-\lambda_{j} \phi_{j}
$$

with $\lambda_{j} \leqslant \lambda_{j+1}$. We also assume that the eigenfunctions are normalized so that their $L^{2}$ norm on $N$ is 1 . We decompose the function $w$ defined on $\left(t_{1}, t_{2}\right) \times N$ as

$$
w(t, y)=\sum_{j \in \mathbb{N}} w_{j}(t) \phi_{j}(y) .
$$

Then $w_{j}$ is a solution of $L_{\lambda_{j}} w_{j}=0$ and the result of Lemma 7.1 implies that $w_{j} \equiv 0$. This completes the proof. q.e.d.

Using similar arguments together with Lemma 7.2 , we also get the: 
Corollary 7.4. Assume that $w$ is a solution of $\mathfrak{L}_{h} w=0$ which is defined on $\mathbb{R} \times N$. Further assume that $w$ is bounded by a constant times $\varphi_{\delta}$ for some $\delta_{ \pm}<\gamma_{ \pm}$. Then $w$ only depends on $t$ and there exists a constant $c \in \mathbb{R}$ such that $w=c w_{\star}$.

Before we proceed, let us observe that the above result holds under a slightly more general assumption, namely that $\delta_{ \pm}<\gamma_{ \pm}\left(\lambda_{1}\right)$, where $\lambda_{1}$ is the first nonzero eigenvalue of the Laplace-Beltrami operator $\Delta_{h}$. Also the function $\varphi_{\delta}$ is the natural extension to $\mathbb{R} \times N$ of the function defined in (7.3).

Proof. Again, we decompose the function $w$ defined on $\mathbb{R} \times N$ as

$$
w(t, y)=\sum_{j \in \mathbb{N}} w_{j}(t) \phi_{j}(y)
$$

Then $w_{j}$ is a solution of $L_{\lambda_{j}} w_{j}=0$ and the result of Lemma 7.2 implies that $w_{j} \equiv 0$, for all $j \neq 0$. When $j=0$, all bounded solutions of $L_{0} w=0$ have to be collinear to $w_{\star}$. This completes the proof. q.e.d.

Let $\Delta$ denote the Laplacian in $\mathbb{R}^{n}$ endowed with the Euclidean metric. We define the elliptic operator

$$
\mathfrak{L}:=-\partial_{t}^{2}-\Delta+\frac{1}{2} W^{\prime \prime}\left(u_{\star}\right) .
$$

The result of Lemma 7.2 also implies the:

Corollary 7.5. Assume that $w \in L^{\infty}\left(\mathbb{R} \times \mathbb{R}^{n}\right)$ is a solution of $\mathfrak{L} w=0$. Then $w$ only depends on $t$ and there exists a constant $c \in \mathbb{R}$ such that $w=c w_{\star}$.

This result seems to be standard and for example appeared (without a proof) in [17]. We have not been able to find a precise reference for it and since this is a key result for the forthcoming argument, we give here a detailed proof.

Proof. We denote by $\mathcal{S}\left(\mathbb{R}^{k}\right)$, the space of smooth rapidly decaying functions which are defined on $\mathbb{R}^{k}$. This space is endowed with the family of semi-norms

$$
[\phi]_{k, l}:=\left\|\left(1+|z|^{k}\right) \nabla^{l} \phi\right\|_{L^{\infty}},
$$

for all $k, l \in \mathbb{N}$, where $z$ denotes the variable in $\mathbb{R}^{k}$. The dual space $\mathcal{S}^{\prime}\left(\mathbb{R}^{k}\right)$ is the space of tempered distributions [20]. 
Let $\mathcal{F}$ denote the Fourier transform in $\mathbb{R}^{n}$ and $\overline{\mathcal{F}}$ the inverse Fourier transform. We define, for all $\phi \in \mathcal{S}\left(\mathbb{R} \times \mathbb{R}^{n}\right)$,

$$
T: \phi \longrightarrow \int_{\mathbb{R}}\langle w(t, \cdot), \overline{\mathcal{F}}(\phi(t, \cdot))\rangle_{\mathcal{S}^{\prime}, \mathcal{S}} d t
$$

This clearly defines a tempered distribution $T \in \mathcal{S}^{\prime}\left(\mathbb{R} \times \mathbb{R}^{n}\right)$. Let us denote by $\xi \in \mathbb{R}^{n}$ the dual variable of $z \in \mathbb{R}^{n}$. Using the fact that $w$ is a solution of $\mathfrak{L} w=0$, we get

$$
\left\langle L_{|\xi|^{2}} T, \Psi\right\rangle_{\mathcal{S}^{\prime}, \mathcal{S}}:=\left\langle T, L_{|\xi|^{2}} \Psi\right\rangle_{\mathcal{S}^{\prime}, \mathcal{S}}=0
$$

for any smooth function $\Psi \in \mathcal{S}\left(\mathbb{R} \times \mathbb{R}^{n}\right)$.

We claim that the support of $T$ is included in $\mathbb{R} \times\{0\}$. Indeed, choose any smooth function $\psi: \mathbb{R} \times \mathbb{R}^{n} \longrightarrow \mathbb{R}$ with compact support in $\mathbb{R} \times\left(\mathbb{R}^{n}-\{0\}\right)$. We define

$$
\begin{aligned}
\Psi(t, \xi):= & \frac{1}{\alpha(\xi)}\left(w_{|\xi|^{2}}^{-}(t) \int_{t}^{+\infty} w_{|\xi|^{2}}^{+}(s) \psi(s, \xi) d s\right. \\
& \left.+w_{|\xi|^{2}}^{+}(t) \int_{-\infty}^{t} w_{|\xi|^{2}}^{-}(s) \psi(s, \xi) d s\right)
\end{aligned}
$$

where

$$
\alpha(\xi):=w_{|\xi|^{2}}^{-}(t) \partial_{t} w_{|\xi|^{2}}^{+}(t)-\partial_{t} w_{|\xi|^{2}}^{-}(t) w_{|\xi|^{2}}^{+}(t),
$$

is the Wronskian of the two independent solutions $w_{|\xi|^{2}}^{ \pm}$of the homogeneous problem $L_{|\xi|^{2}} w=0$ which have been defined at the end of $\S 7.1$ (hence $\alpha$ does not vanish and does not depend on $t$ !). We claim that $L_{|\xi|^{2}} \Psi=\psi$ and also that $\Psi \in \mathcal{S}\left(\mathbb{R} \times \mathbb{R}^{n}\right)$. The first claim follows at once from the fact that $w_{|\xi|^{2}}^{ \pm}$are solutions of $L_{|\xi|^{2}} w=0$. For the second claim, observe that the function $\psi$ has compact support in $\mathbb{R} \times\left(\mathbb{R}^{n}-\{0\}\right)$, hence $\Psi(t, \xi) \equiv 0$ for all $|\xi|$ large enough (say $\left.|\xi| \geqslant c\right)$ and $|\xi|$ small enough (say $|\xi| \leqslant 1 / c$ ). To show that $\Psi$ is rapidly decaying in $t$ when $1 / c \leqslant|\xi| \leqslant c$, we use the fact that, for $\xi \neq 0$, the function $w_{|\xi|^{2}}^{+}$ is exponentially decaying at $+\infty$ and the function $w_{|\xi|^{2}}^{-}$is exponentially decaying at $-\infty$. This implies at once that $\Psi \in \mathcal{S}\left(\mathbb{R} \times \mathbb{R}^{n}\right)$. Therefore, we conclude that

$$
\langle T, \psi\rangle_{\mathcal{S}^{\prime}, \mathcal{S}}=0,
$$

for all $\psi: \mathbb{R} \times \mathbb{R}^{n} \longrightarrow \mathbb{R}$ with compact support in $\mathbb{R} \times\left(\mathbb{R}^{n}-\{0\}\right)$. This proves the claim. 
By a classical result in the theory of distributions [20], we know that $T$ is the linear combination of derivatives (of bounded order), with respect to $t$ and $\xi_{j}, j=1, \ldots, n$, of measures with support on $\mathbb{R} \times\{0\}$. Performing the Fourier transform backward in the $\xi$ variable, we see that the function $w(t, \cdot)$ depends polynomially on the coordinates $z_{j}$ of $z \in \mathbb{R}^{n}$. This, together with the fact that $w$ is bounded in the $z$ variable, implies that the function $w$ only depends on $t$ and hence $w=c w_{\star}$. This completes the proof of the result. q.e.d.

Given $\gamma \in \mathbb{R}$, we define the elliptic operator

$$
\mathfrak{L}_{\gamma}:=-\partial_{t}^{2}-\Delta+\gamma .
$$

Following the proof of Lemma 7.5, we have:

Lemma 7.6. Assume that $\gamma>0$ and that $u \in L^{\infty}\left(\mathbb{R} \times \mathbb{R}^{n}\right)$ is a solution of $\mathfrak{L}_{\gamma} w=0$. Then $w \equiv 0$.

\section{Mapping properties of a model operator}

In this section we study the mapping properties of the operator $\mathfrak{L}_{h}$, given in (7.4), when this operator is defined between weighted Hölder spaces.

\subsection{Function spaces}

Assume that $(N, h)$ is a compact $n$-dimensional compact Riemannian manifold with or without boundary. To begin with, let us define the weighted spaces we will work with:

Definition 8.1. Given $\ell \in \mathbb{N}, \alpha \in(0,1)$ and $\delta:=\left(\delta_{-}, \delta_{+}\right) \in \mathbb{R}^{2}$, we

define the weighted space $\mathcal{C}_{\delta}^{\ell, \alpha}(\mathbb{R} \times N)$ to be the space of functions which are $\ell$ times differentiable, whose $\ell$-th partial derivatives are Hölder of exponent $\alpha$ and for which the weighted norm

$$
\|u\|_{\mathcal{C}_{\delta}^{\ell, \alpha}(\mathbb{R} \times N)}:=\left\|\varphi_{-\delta} u\right\|_{\mathcal{C}^{\ell, \alpha}(\mathbb{R} \times N)},
$$

is finite. Here by definition

$$
\|u\|_{\mathcal{C}^{\ell, \alpha}(\mathbb{R} \times N)}:=\sum_{j=0}^{\ell}\left\|\nabla^{j} u\right\|_{L^{\infty}(\mathbb{R} \times N)}+\sup _{p \neq q \in \mathbb{R} \times N} \frac{\left|\nabla^{\ell} u(p)-\nabla^{\ell} u(q)\right|}{d(p, q)^{\alpha}},
$$

is the standard (unweighted) Hölder norm and $d$ is the geodesic distance in $\mathbb{R} \times N$, for the product metric. 
Roughly speaking, this is the space of functions which, together with their partial derivatives, are bounded by $e^{\delta_{+} t}$ on $(0,+\infty) \times N$ and are bounded by $e^{-\delta_{-} t}$ on $(-\infty, 0) \times N$.

We finally define a 1-codimensional subspace of $\mathcal{C}_{\delta}^{\ell, \alpha}(\mathbb{R} \times N)$.

Definition 8.2. Given $\ell \in \mathbb{N}, \alpha \in(0,1)$ and $\delta:=\left(\delta_{-}, \delta_{+}\right) \in \mathbb{R}^{2}$ such that $\delta_{ \pm}<\gamma_{ \pm}$. The space $\mathcal{D}_{\delta}^{\ell, \alpha}(\mathbb{R} \times N)$ is defined to be the closed subspace of functions $u \in \mathcal{C}_{\delta}^{\ell, \alpha}(\mathbb{R} \times N)$ which are $L^{2}$ orthogonal to $w_{\star}$, i.e.,

$$
\int_{\mathbb{R} \times N} u(t, y) w_{\star}(t) d t d a_{h}=0 .
$$

Naturally, this space is endowed with the induced norm.

The restriction $\delta_{ \pm}<\gamma_{ \pm}$is needed to ensure the convergence of the integral in (8.1), i.e., that $u w_{\star} \in L^{1}(\mathbb{R} \times N)$.

In the case where $N$ has a boundary, we define

$$
\left[\mathcal{D}_{\delta}^{\ell, \alpha}(\mathbb{R} \times N)\right]_{0},
$$

to be the subspace of functions of $\mathcal{D}_{\delta}^{\ell, \alpha}(\mathbb{R} \times N)$ which have 0 Neumann boundary condition on $\mathbb{R} \times \partial N$. In the subsequent sections, it will be convenient to adopt the notation $\left[\mathcal{D}_{\delta}^{\ell, \alpha}(\mathbb{R} \times N)\right]_{0}$ for $\mathcal{D}_{\delta}^{\ell, \alpha}(\mathbb{R} \times N)$ even when $N$ has no boundary, being understood that the condition on the boundary data is void in this latter case.

\subsection{Mapping properties}

Recall that we have defined on the product space $\mathbb{R} \times N$ the partial differential operator

$$
\mathfrak{L}_{h}:=-\partial_{t}^{2}-\Delta_{h}+\frac{1}{2} W^{\prime \prime}\left(u_{\star}\right),
$$

where $\Delta_{h}$ is the Laplace-Beltrami operator on $(N, h)$. We now assume that $(N, h)$ is at least $\mathcal{C}^{1, \alpha}(N)$. This means that, for all $y \in N$, there exists local coordinate $\left(z_{1}, \ldots, z_{n}\right) \in \mathbb{R}^{n}$ (or in $\mathbb{R}_{+}^{n}:=\left\{\left(z_{1}, \ldots, z_{n}\right) \in\right.$ $\left.\mathbb{R}^{n}: z_{n}>0\right\}$ in the case where $\left.y \in \partial N\right)$ in some neighborhood of $y$ for which the coefficients $h_{i j}$ of the metric

$$
h:=\sum_{i, j} h_{i j} d z_{i} \otimes d z_{j},
$$


are $\mathcal{C}^{1, \alpha}$ functions. Clearly, the operator

$$
\mathfrak{L}_{h}:\left[\mathcal{D}_{\delta}^{2, \alpha}(\mathbb{R} \times N)\right]_{0} \longrightarrow \mathcal{D}_{\delta}^{0, \alpha}(\mathbb{R} \times N),
$$

is well-defined and bounded, for any $\delta \in \mathbb{R}^{2}$. It is well known that the mapping properties of the above defined operator depends crucially on the choice of the weight parameter $\delta$. Indeed, the set of indicial roots of $\mathfrak{L}_{h}$ at $+\infty($ resp. $-\infty)$ is defined by $\mathcal{I}_{+}\left(\right.$resp. $\left.\mathcal{I}_{-}\right)$where

$$
\mathcal{I}_{ \pm}:=\left\{\gamma_{ \pm}\left(\lambda_{j}\right): j \geqslant 0\right\}
$$

and $\lambda_{j}$ are the eigenvalues of $\Delta_{h}$ on $N$. Now, if $\delta_{ \pm} \notin \mathcal{I}_{ \pm}$, the operator (8.3) can be shown to have closed range and to be Fredholm. While, if $\delta_{-}$or $\delta_{+}$is an indicial root, then the operator (8.3) does not have close range, and hence is not Fredholm.

The result of Corollary 7.4 yields the injectivity of the operator $\mathfrak{L}_{h}$ when $\delta_{ \pm}<\gamma_{ \pm}$. This, together with a "duality argument" implies that the operator (8.3) is surjective provided $\delta_{ \pm}>-\gamma_{ \pm}$and $\delta_{ \pm} \notin \mathcal{I}_{ \pm}$ (the duality argument does hold stricto sensu when the operator is defined between weighted Lebesgue spaces and the corresponding result in weighted Hölder spaces is then obtained through elliptic regularity theory). In particular, the operator (8.3) is an isomorphism if the weight $\delta_{ \pm} \in\left(-\gamma_{ \pm}, \gamma_{ \pm}\right)$. Most of these mapping properties of $\mathfrak{L}_{h}$ will not be needed. Indeed, we will only need the latter claim on the range of weights for which the operator is an isomorphism. Therefore, in the next Proposition, we concentrate on the proof of this fact.

In addition, we will also show that the inverse of $\mathfrak{L}_{h}$ is bounded independently of the choice of the metric $h$ on $N$, provided an uniform ellipticity condition is fulfilled. Hence, we now assume that there exists $\Lambda>0$ such that:

(i) For all $y \in N$, there exists a local chart

$$
Y_{y}: B^{n}(\Lambda) \longrightarrow N
$$

where $B^{n}(\Lambda)$ denotes the ball or radius $\Lambda$ centered at the origin in $\mathbb{R}^{n}$ (resp. $Y_{y}$ is defined on $B_{+}^{n}(\Lambda)$ the half ball of radius $\Lambda$ centered at the origin in $\mathbb{R}_{+}^{n}$, if $\left.y \in \partial N\right)$.

(ii) For all $z \in B^{n}(\Lambda)$ (or all $z \in B_{+}^{n}(\Lambda)$ ), the metric $h$ at the point of coordinates $z$ is given by

$$
h:=\sum_{i, j} h_{i j}(z) d z_{i} \otimes d z_{j}
$$


where the coefficients $h_{i j}$ are of class $\mathcal{C}^{1, \alpha}$ and satisfy $h_{i j}(0)=\delta_{i}^{j}$.

(iii) For all $z \in B^{n}(\Lambda)$ (or all $z \in B_{+}^{n}(\Lambda)$ ), the metric $h$ satisfies

$$
\sum_{i, j} h^{i j}(z) \xi_{i} \xi_{j} \geqslant \Lambda|\xi|^{2}
$$

for all $\xi \in \mathbb{R}^{n}$.

(iv) We have

$$
\|h\|_{\mathcal{C}^{1, \alpha}(N)} \leqslant \frac{1}{\Lambda}
$$

where the $\mathcal{C}^{1, \alpha}$ norm is the usual one, once a section of the symmetric 2 tensors, is fixed as in (8.5).

The second condition will ensure that the Laplace-Beltrami operator on $N$ is uniformly elliptic while the third condition will ensure uniform Hölder estimates, independent of the choice of the metric.

We prove the following:

Proposition 8.3. Assume that $h$ satisfies (8.6) and (8.7). Further assume that $\delta_{ \pm} \in\left(-\gamma_{ \pm}, \gamma_{ \pm}\right)$. Then, the operator $\mathfrak{L}_{h}$ defined in (8.3) is an isomorphism and there exists a constant $c>0$, only depending on $\Lambda$, such that, for all $w \in\left[\mathcal{D}_{\delta}^{2, \alpha}(\mathbb{R} \times N)\right]_{0}$ we have

$$
\|w\|_{\mathcal{C}_{\delta}^{2, \alpha}(\mathbb{R} \times N)} \leqslant c\left\|\mathfrak{L}_{h} w\right\|_{\mathcal{C}_{\delta}^{0, \alpha}(\mathbb{R} \times N)} .
$$

Proof. As already mentioned, the injectivity of the operator $\mathfrak{L}_{h}$ follows at once from Corollary 7.4. Indeed, given the range in which we have chosen $\delta_{ \pm}$, any solution of $\mathfrak{L}_{h} w=0$ which is bounded by $\varphi_{\delta}$ has to be collinear to $w_{\star}$. Since functions in $\left[\mathcal{D}_{\delta}^{2, \alpha}(\mathbb{R} \times N)\right]_{0}$ are orthogonal to $w_{\star}$ in $L^{2}(\mathbb{R} \times N)$, we conclude that $w \equiv 0$.

We now prove that the operator $\mathfrak{L}_{h}$ defined in (8.3) is surjective. To this aim, we decompose any function $f \in \mathcal{D}_{\delta}^{0, \alpha}(\mathbb{R} \times N)$ as

$$
f(t, y)=f_{0}(t)+\tilde{f}(t, y)
$$

where, for each $t$, the function $\widetilde{f}(t, \cdot)$ is orthogonal to the constant function 1 in $L^{2}(N)$. The proof is now decomposed into 5 steps.

Step 1. We define

$$
w_{0}(t):=w_{\star}(t)\left(\alpha_{0}+\int_{0}^{t} w_{\star}^{-2}(s) \int_{-\infty}^{s} w_{\star}(r) f_{0}(r) d r d s\right),
$$


where the constant $\alpha_{0} \in \mathbb{R}$ is chosen so that

$$
\int_{\mathbb{R}} w_{0} w_{\star} d t=0
$$

Using the fact that $w_{\star} \sim e^{\gamma_{-} t}$ at $-\infty$, together with $-\gamma_{-}<\delta_{-}<\gamma_{-}$, we conclude that

$$
\sup _{(-\infty, 0]} e^{\delta_{-} t}\left|w_{0}\right| \leqslant c_{0} \sup _{\mathbb{R}} e^{\delta_{-} t}\left|f_{0}\right| .
$$

Since $f \in \mathcal{D}_{\delta}^{\ell-2, \alpha}(\mathbb{R} \times N)$, we have

$$
\int_{\mathbb{R}} f_{0} w_{\star} d t=0 .
$$

Therefore, we have the alternative definition of $w_{0}$ as

$$
w_{0}(t):=w_{\star}(t)\left(\alpha_{0}-\int_{0}^{t} w_{\star}^{-2}(s) \int_{s}^{+\infty} w_{\star}(r) f_{0}(r) d r d s\right),
$$

and, using this time the fact that $w_{\star} \sim e^{-\gamma_{+} t}$ at $+\infty$ together with $-\gamma_{+}<\delta_{+}<\gamma_{+}$, we conclude that

$$
\sup _{\mathbb{R}} e^{-\delta_{+} t}\left|w_{0}\right| \leqslant c_{0} \sup _{\mathbb{R}} e^{-\delta_{+} t}\left|f_{0}\right|,
$$

for some constant $c_{0}>0$ which does not depend on $f_{0}$.

Step 2. Making use of the result of Corollary 7.3, we can solve, for each $T>0$

$$
\mathfrak{L}_{h} \widetilde{w}_{T}=\widetilde{f}, \quad \text { in } \quad(-T, T) \times N,
$$

with $\widetilde{w}_{T}=0$ on $\{ \pm T\} \times N$ and 0 Neumann boundary data on $(-T, T) \times$ $\partial N$ if $N$ has a boundary. The superscript $\sim$ is meant to recall that we are working with functions $(t, y) \rightarrow \widetilde{g}(t, y)$ for which the function $\widetilde{g}(t, \cdot)$ is for each $t$ orthogonal to the constant function 1 in $L^{2}(N)$.

Step 3. Choose $t_{0}>0$ large enough so that

$$
\mathfrak{p}:=\min \left(\inf _{\left(-\infty,-t_{0}\right)}\left(\frac{1}{2} W^{\prime \prime}\left(u_{\star}\right)-\delta_{-}^{2}\right), \inf _{\left(t_{0},+\infty\right)}\left(\frac{1}{2} W^{\prime \prime}\left(u_{\star}\right)\right)-\delta_{+}^{2}\right)>0,
$$

then, the potential in the operator $\mathfrak{L}_{h}$ is bounded from below by $\mathfrak{p}$ in $\left(\mathbb{R}-\left[-t_{0}, t_{0}\right]\right) \times N$ and hence $\mathfrak{L}_{h}$ satisfies the maximum principle in this set. Moreover, we have

$$
\mathfrak{L}_{h} e^{-\delta_{-} t}=\left(\frac{1}{2} W^{\prime \prime}\left(u_{\star}\right)-\delta_{-}^{2}\right) e^{-\delta_{-} t} \geqslant \mathfrak{p} e^{-\delta_{-} t},
$$


in $\left(-\infty,-t_{0}\right) \times N$ and

$$
\mathfrak{L}_{h} e^{\delta_{+} t}=\left(\frac{1}{2} W^{\prime \prime}\left(u_{\star}\right)-\delta_{+}^{2}\right) e^{\delta_{+} t} \geqslant \mathfrak{p} e^{\delta_{+} t},
$$

in $\left(t_{0},+\infty\right) \times N$. Hence, the function $t \rightarrow e^{\delta_{+} t}$ (resp. $\left.t \rightarrow e^{-\delta_{-} t}\right)$ can be used as barrier in $\left(t_{0},+\infty\right) \times N$ (resp. in $\left.\left(-\infty, t_{0}\right) \times N\right)$ to prove, for all $T>t_{0}$, the inequality

$$
\begin{aligned}
& \left\|\varphi_{-\delta} \widetilde{w}\right\|_{L^{\infty}((-T, T) \times N)} \\
& \leqslant c\left(\left\|\varphi_{-\delta} \widetilde{w}\right\|_{L^{\infty}\left(\left[-t_{0}, t_{0}\right] \times N\right)}+\left\|\varphi_{-\delta} \widetilde{f}\right\|_{L^{\infty}((-T, T) \times N)}\right),
\end{aligned}
$$

for some constant $c>0$ which only depends on $\delta$ and on $\mathfrak{p}$.

Step 4. We claim that

$$
\left\|\varphi_{-\delta} \widetilde{w}_{T}\right\|_{L^{\infty}((-T, T) \times N)} \leqslant c\left\|\varphi_{-\delta} \tilde{f}\right\|_{L^{\infty}(\mathbb{R} \times N)},
$$

for some constant $c>0$ which depends on $\Lambda$ but neither depends on $T \geqslant 1$ nor on $\widetilde{f}$. We argue by contradiction and assume that there exists a sequence of metrics $h_{k}$ satisfying (8.6) and (8.7), a sequence $T_{k} \geqslant 1$, a sequence of functions $\widetilde{f}_{k}$, and a sequence of solutions of $\mathfrak{L}_{h} \widetilde{w}_{k}=\widetilde{f}_{k}$ in $\left(-T_{k}, T_{k}\right) \times N$, with $\widetilde{w}_{k}=0$ on $\left\{ \pm T_{k}\right\} \times N$ and 0 Neumann boundary data on $\left(-T_{k}, T_{k}\right) \times \partial N$ if $N$ has a boundary, such that

$$
\left\|\varphi_{-\delta} \widetilde{w}_{k}\right\|_{L^{\infty}\left(\left(-T_{k}, T_{k}\right) \times N\right)}=1, \quad \text { and } \quad \lim _{k \rightarrow \infty}\left\|\varphi_{-\delta} \widetilde{f}_{k}\right\|_{L^{\infty}(\mathbb{R} \times N)}=0 .
$$

Furthermore, $\widetilde{f}(t, \cdot)$ and $\widetilde{w}(t, \cdot)$ are, for all $t$, orthogonal to 1 in $L^{2}(N)$.

Observe that the claim is certainly true when $T_{k}$ remains bounded, hence we may well assume that the sequence $T_{k} \geqslant t_{0}$ tends to $+\infty$. Since both $\widetilde{w}_{k}$ and $\widetilde{f}_{k}$ are uniformly bounded on compact subsets, we deduce from elliptic estimates that the sequence of functions $\widetilde{w}_{k}$ is uniformly bounded in $\mathcal{C}^{1, \alpha}$ topology on any compact of $\mathbb{R} \times N$. Now, Ascoli's Theorem together with a standard diagonal argument implies that, up to a subsequence and for some $\alpha^{\prime}<\alpha$, the sequence of metrics $h_{k}$ converges (in $\mathcal{C}^{1, \alpha^{\prime}}(N)$ topology) to $h_{\infty}$, the sequence of functions $\widetilde{w}_{k}$ converges (in $\mathcal{C}^{1, \alpha^{\prime}}(N)$ topology) to $\widetilde{w}_{\infty}$. Passing to the limit in the equation satisfied by $\widetilde{w}_{k}$, we conclude that $\widetilde{w}_{\infty}$ is a weak solution of $\mathfrak{L}_{h_{\infty}} \widetilde{w}_{\infty}=0$ in $\mathbb{R} \times N$, which is bounded by $\varphi_{\delta}$. But Corollary 7.4 and the choice of the parameter $\delta_{ \pm}$in $\left(-\gamma_{ \pm}, \gamma_{ \pm}\right)$imply that $\widetilde{w}_{\infty}=c w_{\star}$, for some constant $c \in \mathbb{R}$. Since the function $\widetilde{w}_{\infty}(t, \cdot)$ is by construction orthogonal to 1 in $L^{2}(N)$, we conclude that $c=0$, hence $\widetilde{w}_{\infty} \equiv 0$. 
Finally, (8.8) implies that, for each $k \in \mathbb{N}$ sufficiently large (so that $\left.T_{k} \geqslant t_{0}\right)$ we have

$$
\begin{aligned}
& \left\|\varphi_{-\delta} \widetilde{w}_{k}\right\|_{L^{\infty}\left(\left[-T_{k}, T_{k}\right] \times N\right)} \\
& =1 \leqslant c\left(\left\|\varphi_{-\delta} \widetilde{w}_{k}\right\|_{L^{\infty}\left(\left[-t_{0}, t_{0}\right] \times N\right)}+\left\|\varphi_{-\delta} \widetilde{f}_{k}\right\|_{L^{\infty}(\mathbb{R} \times N)}\right) .
\end{aligned}
$$

Passing to the limit as $k$ tends to $\infty$, we conclude that

$$
1 \leqslant c\left\|\varphi_{-\delta} \widetilde{w}_{\infty}\right\|_{L^{\infty}\left(\left[-t_{0}, t_{0}\right] \times N\right)},
$$

which implies that $\widetilde{w}_{\infty}$ is not identically equal to 0 . A contradiction. This ends the proof of the claim.

Step 5. Using the results of Steps 2 and 4, standard elliptic estimates and Ascoli's Theorem, we can pass to the limit as $T$ tends to $+\infty$ in the sequence $\widetilde{w}_{T}$ and obtain a function $\widetilde{w}$ solution of $\mathfrak{L}_{h} \widetilde{w}=\widetilde{f}$ in $\mathbb{R} \times N$. Furthermore, the result of Step 4 implies that

$$
\left\|\varphi_{-\delta} \widetilde{w}\right\|_{L^{\infty}(\mathbb{R} \times N)} \leqslant c\left\|\varphi_{-\delta} \widetilde{f}\right\|_{L^{\infty}(\mathbb{R} \times N)}
$$

for some constant $c>0$ which only depends on $\Lambda$. Collecting this result and the result of Step 1, we conclude that $w=w_{0}+\widetilde{w}$ is a solution of $\mathfrak{L}_{h} w=f$ which satisfies (8.1). Furthermore, using Schauder's estimates, we also conclude that

$$
\|w\|_{\mathcal{C}_{\delta}^{2, \alpha}(\mathbb{R} \times N)} \leqslant c\|f\|_{\mathcal{C}_{\delta}^{0, \alpha}(\mathbb{R} \times N)},
$$

for some constant $c>0$ which only depends on $\Lambda$. In particular $w \in$ $\left[\mathcal{D}_{\delta}^{2, \alpha}(\mathbb{R} \times N)\right]_{0}$. This completes the proof of the result. $\quad$ q.e.d.

Once Proposition 8.3 is proven, it is easy to see that:

Proposition 8.4. Assume that $\delta_{ \pm}<\gamma_{ \pm}$. If $w \in\left[\mathcal{D}_{\delta}^{2, \alpha}(\mathbb{R} \times N)\right]_{0}$ and $f \in \mathcal{D}_{\delta}^{0, \alpha}(\mathbb{R} \times N)$ satisfy $\mathfrak{L}_{h} w=f$ and if in addition

$$
\int_{\mathbb{R}} f(t, y) w_{\star}(t) d t=0,
$$

for all $y \in N$, then

$$
\int_{\mathbb{R}} w(t, y) w_{\star}(t) d t=0,
$$

for all $y \in N$. 
Proof. Recall that $\left(\phi_{j}, \lambda_{j}\right)_{j \geqslant 0}$ are the eigendata of $\Delta_{h}$ (with $0 \mathrm{Neu}-$ mann boundary condition if $\partial N$ is not empty). For all $j \geqslant 1$, we multiply the equation $\mathfrak{L}_{h} w=f$ by $w_{\star} \phi_{j}$ and integrate by parts over $\mathbb{R} \times N$. We obtain

$$
\int_{\mathbb{R} \times N} w \mathfrak{L}_{h}\left(\phi_{j} w_{\star}\right) d t d a_{h}=0
$$

Since $\mathfrak{L}_{h}\left(w_{\star} \phi_{j}\right)=\lambda_{j} w_{\star} \phi_{j}$, we conclude that

$$
\lambda_{j} \int_{N} \phi_{j}\left(\int_{\mathbb{R}} w w_{\star} d t\right) d a_{h}=0
$$

When $j \neq 0, \lambda_{j} \neq 0$, hence this implies that the function

$$
\Phi(y):=\int_{\mathbb{R}} w(t, y) w_{\star}(t) d t
$$

is orthogonal to $\phi_{j}$ in $L^{2}(N)$. By construction this function is also orthogonal to $\phi_{0}$, which is the constant function, in $L^{2}(N)$. Since the $\left(\phi_{j}\right)_{j \geqslant 0}$ form a Hilbert basis of $L^{2}(N)$, we conclude that $\Phi \equiv 0$. q.e.d.

Before we proceed further, let us comment on our choice of function spaces. Observe that, in (8.3), we could have replaced the spaces $\mathcal{D}_{\delta}^{\ell, \alpha}(\mathbb{R} \times N)$ by the spaces $\mathcal{C}_{\delta}^{\ell, \alpha}(\mathbb{R} \times N)$ and we could have defined

$$
\widetilde{\mathfrak{L}}_{h}:\left[\mathcal{C}_{\delta}^{2, \alpha}(\mathbb{R} \times N)\right]_{0} \longrightarrow \mathcal{C}_{\delta}^{0, \alpha}(\mathbb{R} \times N)
$$

Then all the above results about the set of weights for which the operator is Fredholm remain true. However, this time, the injectivity of $\widetilde{\mathfrak{L}}_{h}$ only holds provided $\delta_{ \pm} \lesssim-\gamma_{ \pm}$and, using a "duality argument", this implies that the operator $\widetilde{\mathfrak{L}}_{h}$ is now surjective when $\delta_{ \pm}>\gamma_{ \pm}$are not indicial roots. Hence, this choice of function spaces would force us to work in a space of functions which blow up exponentially at $\pm \infty$ and this would not be suitable for the forthcoming nonlinear analysis. 


\section{Mapping properties of a singularly perturbed linear operator}

\subsection{Function spaces}

Assume that $(N, h)$ is a $n$-dimensional compact Riemannian manifold, with or without boundary and further assume that the metric $h$ on $N$ satisfies (8.6) and (8.7), for some fixed constant $\Lambda>0$. We now turn to the study of the operator

$$
\mathfrak{L}_{\varepsilon}:=-\varepsilon^{2}\left(\partial_{t}^{2}+\Delta_{h}\right)+\frac{1}{2} W^{\prime \prime}\left(u_{\star}(\cdot / \varepsilon)\right),
$$

where $\varepsilon \in(0,1)$ is a fixed parameter. This operator still depends on $h$ but, since we now focus our attention on its dependence with respect to the parameter $\varepsilon$, we omit the subscript $h$. Taking the parameter $\varepsilon$ into account, we now define:

Definition 9.1. Given $\ell \in \mathbb{N}, \alpha \in(0,1), \delta:=\left(\delta_{-}, \delta_{+}\right) \in \mathbb{R}^{2}$ and $\varepsilon \in(0,1)$, we define the weighted space $\mathcal{C}_{\delta, \varepsilon}^{\ell, \alpha}(\mathbb{R} \times N)$ to be the space of functions which are $\ell$ times differentiable, whose $\ell$-th partial derivaties are Hölder of exponent $\alpha$ and for which the weighted norm

$$
\|u\|_{\mathcal{C}_{\delta, \varepsilon}^{\ell, \alpha}(\mathbb{R} \times N)}:=\left\|\varphi_{-\delta}(\cdot / \varepsilon) u\right\|_{\mathcal{C}_{\varepsilon}^{\ell, \alpha}(\mathbb{R} \times N)},
$$

is finite. Here, by definition

$$
\|u\|_{\mathcal{C}_{\varepsilon}^{\ell, \alpha}(\mathbb{R} \times N)}:=\sum_{j=0}^{\ell} \varepsilon^{j}\left\|\nabla^{j} u\right\|_{L^{\infty}(\mathbb{R} \times N)}+\varepsilon^{\ell+\alpha}\left\|\nabla^{\ell} u\right\|_{\mathcal{C}^{0, \alpha}(\mathbb{R} \times N)} .
$$

When $\delta_{ \pm}<\gamma_{ \pm}$, we also define the spaces $\mathcal{D}_{\delta, \varepsilon}^{\ell, \alpha}(\mathbb{R} \times N)$ as the 1 codimensional subspace of functions $u \in \mathcal{C}_{\delta, \varepsilon}^{\ell, \alpha}(\mathbb{R} \times N)$ which are $L^{2}$ orthogonal to the function $w_{\star}(\cdot / \varepsilon)$. For fixed $\varepsilon$ the results of $\S 8$ hold and this shows that

$$
\mathfrak{L}_{\varepsilon}:\left[\mathcal{D}_{\delta, \varepsilon}^{2, \alpha}(\mathbb{R} \times N)\right]_{0} \longrightarrow \mathcal{D}_{\delta, \varepsilon}^{0, \alpha}(\mathbb{R} \times N),
$$

is an isomorphism, provided $\delta_{ \pm} \in\left(-\gamma_{ \pm}, \gamma_{ \pm}\right)$. Our aim is now to understand the mapping properties of $\mathfrak{L}_{\varepsilon}$ as the scaling parameter $\varepsilon$ tends to 0 . Unfortunately, if we work with the function spaces $\mathcal{D}_{\delta, \varepsilon}^{2, \alpha}(\mathbb{R} \times N)$, the norm of the inverse of the above defined operator blows up as $\varepsilon$ tends to 0 . 
To get a better understanding of the underlying phenomena, we digress slightly and study the spectrum of the self-adjoint operator $\mathfrak{L}_{\varepsilon}$ acting on functions which are defined on $[-1,1] \times N$, have 0 Dirichlet boundary conditions on $\{ \pm 1\} \times N$ and 0 Neumann boundary conditions on $[-1,1] \times \partial N$ if $\partial N$ is not empty. Recall that there is an eigenfunction decomposition of the functions $w$ defined on $[-1,1] \times N$ as

$$
w(t, y)=\sum_{j=0}^{\infty} w_{j}(t) \phi_{j}(y),
$$

where $\left(\phi_{j}, \lambda_{j}\right)$ are the eigendata of $\Delta_{h}$. This splitting induces a splitting of the operator $\mathfrak{L}_{\varepsilon}$ into a sequence of second order ordinary differential operators

$$
L_{\varepsilon, j}:=-\varepsilon^{2} \partial_{t}^{2}+\varepsilon^{2} \lambda_{j}+\frac{1}{2} W^{\prime}\left(u_{\star}(\cdot / \varepsilon)\right),
$$

acting on functions defined on $[-1,1]$. The spectrum of $\mathfrak{L}_{\varepsilon}$ is then the union of the spectra of the operators $L_{\varepsilon, j}$. Namely

$$
\operatorname{Spec}\left(\mathfrak{L}_{\varepsilon}\right)=\bigcup_{j \in \mathbb{N}} \operatorname{Spec}\left(L_{\varepsilon, j}\right) .
$$

Furthermore, the spectrum of $L_{\varepsilon, j}$ is equal to the spectrum of $L_{\varepsilon, 0}$ shifted by $\varepsilon^{2} \lambda_{j}$. Now, the spectrum of $L_{\varepsilon, 0}$ is given by

$$
\operatorname{Spec} L_{\varepsilon, 0}:=\left\{\mu_{0, \varepsilon}<\mu_{1, \varepsilon}<\mu_{2, \varepsilon} \cdots\right\} .
$$

All the eigenvalues are simple since the operator is a second order ordinary differential operator. Moreover, arguing as in the proof of Lemma 7.1, one can show that $\mu_{0, \varepsilon}>0$. The existence of the function $w_{\star}$ also implies easily that $\mu_{0, \varepsilon}$ decays exponentially fast to 0 as $\varepsilon$ tends to 0 . Furthermore, if $w_{0, \varepsilon}$ denote the eigenfunctions associated to $\mu_{0, \varepsilon}$, which are normalized so that $w_{0, \varepsilon}(0)=1$, the sequence of rescaled functions $\left(w_{0, \varepsilon}(\varepsilon \cdot)\right)_{\varepsilon>0}$ converges on compacts to $w_{\star}$, as $\varepsilon$ tends to 0 . Concerning the second eigenvalue $\mu_{1, \varepsilon}$, one can show that

$$
\lim _{\varepsilon \rightarrow 0} \mu_{1, \varepsilon}>0
$$

To prove this fact, one can argue by contradiction as in the proof of Proposition 8.3. Since these results are not needed in our analysis, we leave the proofs to the reader. 
On a heuristic level, the above study shows that, as $\varepsilon$ tends to 0 , the number of small eigenvalues of $\mathfrak{L}_{\varepsilon}$ which are close to 0 tends to $+\infty$. Moreover the corresponding eigenfunctions are of the form $w_{0, \varepsilon} \phi_{j}$, where $w_{0, \varepsilon}(\varepsilon \cdot)$ tends to $w_{\star}$ as $\varepsilon$ tends to 0 . Since we want to work with an operator whose inverse is uniformly bounded as $\varepsilon$ tends to 0 , we need to replace the spaces we work with by much smaller function spaces. It is standard to work orthogonally to all the eigenfunctions which correspond to small eigenvalues to recover an operator whose inverse is uniformly bounded. Since the eigenfunctions corresponding to small eigenvalues tend to $w_{\star}(\cdot / \varepsilon) \phi_{j}$, as $\varepsilon$ tends to 0 , it should be enough to work orthogonally to all the functions of the form $w_{\star}(\cdot / \varepsilon) \phi_{j}$, in order to recover an operator whose inverse is uniformly bounded.

The above should be enough to justify why we replace the spaces $\mathcal{D}_{\delta, \varepsilon}^{\ell, \alpha}(\mathbb{R} \times N)$ by the much smaller function spaces which we now describe.

Definition 9.2. Given, $\ell \in \mathbb{N}, \alpha \in(0,1), \delta:=\left(\delta_{-}, \delta_{+}\right) \in \mathbb{R}^{2}$ and $\varepsilon \in(0,1)$ such that $\delta_{ \pm}<\gamma_{ \pm}$, we define the weighted space $\mathcal{E}_{\delta, \varepsilon}^{\ell, \alpha}(\mathbb{R} \times N)$ to be the space of functions $u \in \mathcal{C}_{\delta, \varepsilon}^{\ell, \alpha}(\mathbb{R} \times N)$, which satisfy

$$
\forall y \in N, \quad \int_{\mathbb{R}} u(t, y) w_{\star}(t / \varepsilon) d t=0 .
$$

This space is endowed with the induced norm.

Observe that we now impose infinitely many constraints on the functions $u$, so $\mathcal{E}_{\delta, \varepsilon}^{\ell, \alpha}(\mathbb{R} \times N)$ has infinite codimension in $\mathcal{C}_{\delta, \varepsilon}^{\ell, \alpha}(\mathbb{R} \times N)$. The restriction $\delta_{ \pm}<\gamma_{ \pm}$is needed to ensure the convergence of the integrals in (9.2).

In the case where $N$ has a boundary, we define $\left[\mathcal{E}_{\delta, \varepsilon}^{\ell, \alpha}(\mathbb{R} \times N)\right]_{0}$, to be the subspace of functions of $\mathcal{E}_{\delta, \varepsilon}^{\ell, \alpha}(\mathbb{R} \times N)$ which have 0 Neumann boundary condition on $\mathbb{R} \times \partial N$. Again, we keep the same notation for this space whether $N$ has a boundary or not.

\subsection{Mapping properties}

It will be interesting to take into account the parameter $\varepsilon$ in order to relax slightly the assumptions on the metric $h$. Indeed, we will still consider that $h$ satisfies (8.6) but instead of (8.7) we now assume that $\ell \geqslant 2$ is fixed and that $h$ satisfies the weaker condition

$$
\|h\|_{\mathcal{C}_{\varepsilon}^{\ell-1, \alpha}(N)} \leqslant \frac{1}{\Lambda}
$$


where by definition

$$
\begin{aligned}
\|h\|_{\mathcal{C}_{\varepsilon}^{\ell, \alpha}(N)}:= & \left\|h_{i j}\right\|_{L^{\infty}(N)}+\sum_{j=1}^{\ell} \varepsilon^{j-1}\left\|\nabla^{j} h_{i j}\right\|_{L^{\infty}(N)} \\
& +\varepsilon^{\ell-1+\alpha}\left\|\nabla^{\ell} h_{i j}\right\|_{\mathcal{C}^{0, \alpha}(N)} .
\end{aligned}
$$

To define these, we use the local charts defined in (8.4) and the sections of the symmetric two tensors defined in (8.5).

Remark 9.3. At first glance, this condition does not seem very natural since, paralleling the definition of the spaces $\mathcal{C}_{\varepsilon}^{\ell, \alpha}(\mathbb{R} \times N)$, it would have been more natural to require that

$$
|h|_{\mathcal{C}_{\varepsilon}^{\ell, \alpha}(N)} \leqslant \frac{1}{\Lambda}
$$

where

$$
|h|_{\mathcal{C}_{\varepsilon}^{\ell, \alpha}(N)}:=\sum_{j=0}^{\ell} \varepsilon^{j}\left\|\nabla^{j} h_{i j}\right\|_{L^{\infty}(N)}+\varepsilon^{\ell+\alpha}\left\|\nabla^{\ell} h_{i j}\right\|_{\mathcal{C}^{0, \alpha}(N)} .
$$

However, as we will see in the proof of the next Proposition, we will need a control on the $\mathcal{C}^{1}$ norm of $h$ which is slightly better then the one which is provided by (9.5).

Indeed, if $\Lambda$ is assumed to be fixed, $\varepsilon \nabla h_{i j}$ converges uniformly to 0 as $\varepsilon$ tends to 0 when $h$ is assumed to satisfy (9.3), while this fact is not guaranteed by (9.5). Now the fact that $\varepsilon \nabla h_{i j}$ converges uniformly to 0 as $\varepsilon$ tends to 0 will be used in the proof of the next Proposition and this will allow us to use the result of Corollary 7.5. It is possible to use (9.5) instead of (9.3) and this simplifies some of the forthcoming statements. However, we would have to pay a price, namely we would have to prove a result similar to Corollary 7.5 when $\Delta$ is replaced by a more general elliptic operator on $\mathbb{R}^{n}$.

Observe that, granted the above definitions, the operator

$$
\mathfrak{L}_{\varepsilon}:\left[\mathcal{E}_{\delta, \varepsilon}^{2, \alpha}(\mathbb{R} \times N)\right]_{0} \longrightarrow \mathcal{E}_{\delta, \varepsilon}^{0, \alpha}(\mathbb{R} \times N),
$$


is well-defined. Indeed, given $w \in \mathcal{E}_{\delta, \varepsilon}^{2, \alpha}(\mathbb{R} \times N)$ we have

$$
\begin{aligned}
\int_{\mathbb{R}} \mathfrak{L}_{\varepsilon} w w_{\star}(\cdot / \varepsilon) d t \\
=\int_{\mathbb{R}}\left(-\varepsilon^{2}\left(\partial_{t}^{2}+\Delta_{h}\right)+\frac{1}{2} W^{\prime \prime}\left(w_{\star}(\cdot / \varepsilon)\right)\right) w w_{\star}(\cdot / \varepsilon) d t \\
=\int_{\mathbb{R}}\left(-\varepsilon^{2} \partial_{t}^{2}+\frac{1}{2} W^{\prime \prime}\left(w_{\star}(\cdot / \varepsilon)\right)\right) w w_{\star}(\cdot / \varepsilon) d t \\
\quad-\varepsilon^{2} \Delta_{h}\left(\int_{\mathbb{R}} w w_{\star}(\cdot / \varepsilon) d t\right) \\
=\int_{\mathbb{R}}\left(-\varepsilon^{2} \partial_{t}^{2}+\frac{1}{2} W^{\prime \prime}\left(w_{\star}(\cdot / \varepsilon)\right)\right) w_{\star}(\cdot / \varepsilon) w d t \\
=0 .
\end{aligned}
$$

Hence, $\mathfrak{L}_{\varepsilon} w \in \mathcal{E}_{\varepsilon}^{0, \alpha}(\mathbb{R} \times N)$. Recall that we have assumed that the metric $h$ satisfies (8.6) and (8.7). This implies that $\mathfrak{L}_{\varepsilon}$ is uniformly bounded as $\varepsilon$ varies in $(0,1)$. Applying the results of $\S 8$, we see that $\mathfrak{L}_{\varepsilon}$, defined in (9.6) is an isomorphism, provided $\delta_{ \pm} \in\left(-\gamma_{ \pm}, \gamma_{ \pm}\right)$. In the next result, we show that the inverse of $\mathfrak{L}_{\varepsilon}$ is also uniformly bounded as $\varepsilon$ varies in $(0,1)$.

Proposition 9.4. Assume that $h$ satisfies (8.6), (9.3) and further assume that $\delta_{ \pm} \in\left(-\gamma_{ \pm}, 0\right]$. There exists a constant $c>0$ (only depending on $\Lambda)$, such that, for all $\varepsilon \in(0,1)$ and for all $w \in\left[\mathcal{E}_{\delta, \varepsilon}^{2, \alpha}(\mathbb{R} \times N)\right]_{0}$, we have

$$
\|w\|_{\mathcal{C}_{\delta, \varepsilon}^{2, \alpha}(\mathbb{R} \times N)} \leqslant c\left\|\mathfrak{L}_{\varepsilon} w\right\|_{\mathcal{C}_{\delta, \varepsilon}^{0, \alpha}(\mathbb{R} \times N)} .
$$

The restriction $\delta_{ \pm} \leqslant 0$ will be needed to apply the result of Corollary 7.5 .

Proof. We claim that, there exists a constant $c>0$, such that if $w$ and $\mathfrak{L}_{\varepsilon} w$ are functions bounded by $\varphi_{\delta}(\cdot / \varepsilon$ ) (and $w$ has 0 Neumann boundary condition on $\mathbb{R} \times \partial N$ if $N$ has a boundary) and if $w$ satisfies (9.2), then

$$
\left\|\varphi_{-\delta}(\cdot / \varepsilon) w\right\|_{L^{\infty}(\mathbb{R} \times N)} \leqslant c\left\|\varphi_{-\delta}(\cdot / \varepsilon) \mathfrak{L}_{\varepsilon} w\right\|_{L^{\infty}(\mathbb{R} \times N)}
$$

As in the proof of Proposition 8.3, the proof of the claim relies on the observation that there exists $t_{0}>0$ and $c>0$ such that, if $w$ and 
$\mathfrak{L}_{\varepsilon} w$ are functions bounded by $\varphi_{\delta}(\cdot / \varepsilon)$ (and $w$ has 0 Neumann boundary condition on $\mathbb{R} \times \partial N$ if $N$ has a boundary), then

$$
\begin{aligned}
& \left\|\varphi_{-\delta}(\cdot / \varepsilon) w\right\|_{L^{\infty}(\mathbb{R} \times N)} \\
& \leqslant c\left(\|w\|_{L^{\infty}\left(\left[-t_{0} \varepsilon, t_{0} \varepsilon\right] \times N\right)}+\left\|\varphi_{-\delta}(\cdot / \varepsilon) \mathfrak{L}_{\varepsilon} w\right\|_{L^{\infty}(\mathbb{R} \times N)}\right) .
\end{aligned}
$$

see (8.8). In order to prove the claim, we argue by contradiction. If the claim were not true, there would exist a sequence $\varepsilon_{k}$ tending to 0 , a sequence of metrics $h_{k}$ satisfying (8.6) and (9.3), a sequence of functions $w_{k}$ satisfying (9.2) such that

$$
\begin{aligned}
& \left\|\varphi_{-\delta}\left(\cdot / \varepsilon_{k}\right) w_{k}\right\|_{L^{\infty}(\mathbb{R} \times N)}=1, \quad \text { and } \\
& \lim _{k \rightarrow \infty}\left\|\varphi_{-\delta}\left(\cdot / \varepsilon_{k}\right) \mathfrak{L}_{\varepsilon_{k}} w_{k}\right\|_{L^{\infty}(\mathbb{R} \times N)}=0 .
\end{aligned}
$$

Moreover, the functions $w_{k}$ have 0 Neumann boundary condition on $\mathbb{R} \times \partial N$ if $N$ has a boundary.

Observe that (9.8) implies that, for each $k \in \mathbb{N}$

$$
1 \leqslant c\left(\left\|\varphi_{-\delta}\left(\cdot / \varepsilon_{k}\right) w_{k}\right\|_{L^{\infty}\left(\left[-t_{0} \varepsilon_{k}, t_{0} \varepsilon_{k}\right] \times N\right)}+\left\|\varphi_{-\delta}\left(\cdot / \varepsilon_{k}\right) \mathfrak{L}_{\varepsilon_{k}} w_{k}\right\|_{L^{\infty}(\mathbb{R} \times N)}\right) .
$$

Furthermore, since the functions $y \longrightarrow \sup _{\left[-t_{0} \varepsilon_{k}, t_{0} \varepsilon_{k}\right]} \varphi_{-\delta}\left(t / \varepsilon_{k}\right)\left|w_{k}(t, y)\right|$ are continuous, for each $k \geqslant 0$, one can choose a point $y_{k} \in N$ such that

$$
\begin{aligned}
1 \leqslant c & \left(\left\|\varphi_{-\delta}\left(\cdot / \varepsilon_{k}\right) w_{k}\left(\cdot, y_{k}\right)\right\|_{L^{\infty}\left(\left[-t_{0} \varepsilon_{k}, t_{0} \varepsilon_{k}\right]\right)}\right. \\
& \left.+\left\|\varphi_{-\delta}\left(\cdot / \varepsilon_{k}\right) \mathfrak{L}_{\varepsilon_{k}} w_{k}\right\|_{L^{\infty}(\mathbb{R} \times N)}\right) .
\end{aligned}
$$

The hypersurface $N$ being compact, we can assume without loss of generality that the sequence $y_{k}$ converges in $N$.

We define, for all $s \in \mathbb{R}$ and for all $z \in \mathbb{R}^{n}$ (or $\mathbb{R}_{+}^{n}$ ) close enough to the origin

$$
\widetilde{w}_{k}(s, \widetilde{z}):=w_{k}\left(\varepsilon_{k} s, Y_{y_{k}}\left(\varepsilon_{k} \widetilde{z}\right)\right) .
$$

In the case where $N$ has a boundary, we define

$$
\rho_{k}:=d\left(y_{k}, \partial N\right) / \varepsilon_{k},
$$

where $d$ denotes the geodesic distance on $N$ for the metric $h_{k}$. We now distinguish a few cases according to the behavior of the sequence $\left(\rho_{k}\right)_{k}$, which can be assumed to converge to $\rho_{\infty} \in[0, \infty]$. 
Case 1. Assume that either $N$ has no boundary or $N$ has a boundary and $\rho_{\infty}=+\infty$. Since both $w_{k}$ and $\mathfrak{L}_{\varepsilon_{k}} w_{k}$ are uniformly bounded on compact sets, we deduce from elliptic estimates that the sequence of functions $\widetilde{w}_{k}$ is bounded in $\mathcal{C}^{1, \alpha}$ topology on any compact of $\mathbb{R} \times \mathbb{R}^{n}$. Now, Ascoli's Theorem together with a standard diagonal argument implies that, up to a subsequence and for some $\alpha^{\prime}<\alpha$ the sequence of functions $\widetilde{w}_{k}$ converge (in $\mathcal{C}^{1, \alpha^{\prime}}$ topology) to $\widetilde{w}_{\infty}$. Furthermore, $w_{\infty}$ is bounded by $\varphi_{\delta}$. Passing to the limit in the equation satisfied by $\widetilde{w}_{k}$, we claim that the function $\widetilde{w}_{\infty}$ is a weak solution of

$$
\left(\partial_{t}^{2}+\Delta-\frac{1}{2} W^{\prime \prime}\left(u_{\star}\right)\right) \widetilde{w}_{\infty}=0
$$

in $\mathbb{R} \times \mathbb{R}^{n}$. This is precisely at this point that we use the full strength of (9.3) which guarantees that the $\mathcal{C}^{1}$ norm of the coefficients of the metrics $h_{k}$ are uniformly bounded, hence the sequence of dilated metrics converges to the flat metric. While the weaker more natural condition given in Remark 9.3 would only have ensured that the sequence of dilated metrics converges to some metric on $\mathbb{R}^{n}$. Indeed, observe that, in order to define the functions $\widetilde{w}_{k}$, we have chosen normal coordinates $\varepsilon_{k} z$ to parameterize $N$ in some neighborhood of the point $y_{k}$. In these coordinates the metric $h_{k}=\varepsilon_{k}^{2} \widetilde{h}_{k}$ where

$$
\widetilde{h}_{k}:=\sum_{i j} \widetilde{h}_{k, i j}(\widetilde{z}) d \widetilde{z}_{i} \otimes d \widetilde{z}_{j}
$$

satisfies $\widetilde{h}_{k, i j}(0)=\delta_{i}^{j}$ and

$$
\left\|\nabla^{m} \widetilde{h}_{k, i j}\right\|_{L^{\infty}} \leqslant c \varepsilon_{k},
$$

for $m=1, \ldots, 2$, thanks to (9.3). Here all norms are evaluated on a fixed compact, $c>0$ only depends on $\Lambda$ and the partial derivatives are taken with respect to the $\widetilde{z}_{i}$. This in turn implies that the Laplace-Beltrami operator $\varepsilon_{k}^{2} \Delta_{h_{k}}$ can be expanded as

$$
\varepsilon_{k}^{2} \Delta_{h_{k}}=\Delta+\mathcal{O}\left(\varepsilon_{k}|\widetilde{z}| \partial_{\widetilde{z}_{i}} \partial_{\widetilde{z}_{j}}\right)+\mathcal{O}\left(\varepsilon_{k} \partial_{\widetilde{z}_{i}}\right) .
$$

This explains why the equation satisfied by $\widetilde{w}_{\infty}$ involves the operator $\Delta$.

Case 2. Assume that $N$ has a boundary and that $\rho_{\infty}<+\infty$. Applying the above argument, we can assume that, up to a subsequence, 
the sequence of functions $\widetilde{w}_{k}$ converges to $\widetilde{w}_{\infty}$. Furthermore, $\widetilde{w}_{\infty}$ is a weak solution of

$$
\left(\partial_{t}^{2}+\Delta-\frac{1}{2} W^{\prime \prime}\left(u_{\star}\right)\right) \widetilde{w}_{\infty}=0
$$

in $\mathbb{R} \times \mathbb{R}_{+}^{n}$ which is bounded by $\varphi_{\delta}$. In addition, $w$ has 0 Neumann boundary data and we can then extend $\widetilde{w}_{\infty}$ to $\mathbb{R} \times \mathbb{R}^{n}$ by reflection across the hyperplane $z_{n}=0$ to reduce to Case 1 .

In either case, Lebesgue's convergence Theorem implies that $\widetilde{w}_{\infty}$ satisfies

$$
\int_{\mathbb{R} \times \mathbb{R}^{n}} \widetilde{w}_{\infty} w_{\star} d t d z=0 .
$$

We give the proof of this fact when $\rho_{\infty}=+\infty$, obvious modifications are needed to handle the case where $\rho_{\infty}<+\infty$. Given a function $\eta: \mathbb{R}^{n} \longrightarrow \mathbb{R}$, with compact support we use the fact that, for all $z$ in $\mathbb{R}^{n}$ and all $k$ large enough

$$
\int_{\mathbb{R}} \widetilde{w}_{k}(s, z) w_{\star}(s) d s=0 .
$$

Provided $k$ is chosen large enough so that the domain of definition of $z \rightarrow \widetilde{w}_{k}(s, z)$ includes the support of $\eta$, we multiply this identity by $\eta$ and integrate over $z$ to get

$$
\int_{\mathbb{R} \times \mathbb{R}^{n}} \eta(z) \widetilde{w}_{k}(s, z) w_{\star}(s) d s d a_{h_{k}}=0 .
$$

Passing to the limit as $k$ tends to $\infty$, we conclude that

$$
\int_{\mathbb{R} \times \mathbb{R}^{n}} \eta(z) \widetilde{w}_{\infty}(s, z) w_{\star}(s) d s d z=0 .
$$

This identity being valid for all $\eta$, we finally obtain, for all $z \in \mathbb{R}^{n}$

$$
\int_{\mathbb{R}} \widetilde{w}_{\infty}(s, z) w_{\star}(s) d s=0 .
$$

By construction, the function $\widetilde{w}_{\infty}$ is bounded by $\varphi_{\delta}$ and since we have assumed that $\delta_{ \pm} \leqslant 0$, this implies that $\widetilde{w}_{\infty}$ is bounded. We now apply the result of Corollary 7.5 which shows that $\widetilde{w}_{\infty}$ only depends on $t$. Since all bounded solutions of $L_{0} w=0$ are collinear to $w_{\star}$, we can 
write $\widetilde{w}_{\infty}(s, z)=c w_{\star}(s)$ for some constant $c \in \mathbb{R}$, and (9.10) implies that $c=0$. Therefore, we have shown that $\widetilde{w}_{\infty} \equiv 0$.

Finally, (9.9) implies that, for each $k \in \mathbb{N}$

$$
1 \leqslant c\left(\left\|\varphi_{-\delta} \widetilde{w}_{k}(\cdot, 0)\right\|_{L^{\infty}\left(\left[-t_{0}, t_{0}\right] \times N\right)}+\left\|\varphi_{-\delta}\left(\cdot / \varepsilon_{k}\right) \mathfrak{L}_{\varepsilon_{k}} w_{k}\right\|_{L^{\infty}(\mathbb{R} \times N)}\right),
$$

passing to the limit as $k$ tends to $\infty$ in this inequality, we conclude that

$$
1 \leqslant c\left\|\varphi_{-\delta} \widetilde{w}_{\infty}(\cdot, 0)\right\|_{L^{\infty}\left(\left[-t_{0}, t_{0}\right] \times N\right)}
$$

which implies that $\widetilde{w}_{\infty}$ is not identically equal to 0 . Since we have reached a contradiction, the proof of the claim is complete.

The claim being proved the result of the Proposition follows immediately from Schauder's estimates which are applied in geodesic balls of radius $\varepsilon$ in $\mathbb{R} \times N$.

q.e.d.

Obviously, this result could have been obtained directly without any reference to the result of Proposition 8.3. However, we feel that the decomposition of the proof into two different steps sheds light on the choice of the function spaces and in particular explains where the conditions (8.1) and (9.2) enter into play.

\section{Fermi coordinates}

Assume that $N \subset M$ is an admissible hypersurface. To begin with, let us assume that $N$ has no boundary. We will explain in the last paragraph of this section the modifications which are needed to handle the case where the boundary of $N$ is not empty.

For any $p \in M$ we define $d_{N}(p)$ to be the signed geodesic distance from $p$ to $N$. This means that $d_{N}(p)$ is the geodesic distance from $p$ to $N$ if $p \in M^{+}(N)$ and that $d_{N}(p)$ is equal to minus the geodesic distance from $p$ to $N$ if $p \in M^{-}(N)$. We define

$$
V_{\tau}(N):=\left\{p \in M: d_{N}(p) \in(-\tau, \tau)\right\}
$$

It is well-known that for all $\tau$ small enough, the set $V_{\tau}(N)$ is a tubular neighborhood of $N$ in $M$, provided $N$ is at least $\mathcal{C}^{2}$. In order to measure the regularity of a function $u$ which is defined in $V_{\tau}(N)$, we define for 


$$
\begin{aligned}
& \ell \geqslant 0, \alpha \in(0,1) \text { and } \varepsilon \in(0,1) \\
& \qquad u\left\|_{\mathcal{C}_{\varepsilon}^{\ell, \alpha}\left(V_{\tau}(N)\right)}:=\sum_{j=0}^{\ell} \varepsilon^{j}\right\| \nabla^{j} u \|_{L^{\infty}\left(V_{\tau}(N)\right)} \\
& \quad+\varepsilon^{\ell+\alpha} \sup _{p \neq q \in V_{\tau}(N)} \frac{\left|\nabla^{\ell} u(p)-\nabla^{\ell} u(q)\right|}{d(p, q)^{\alpha}}
\end{aligned}
$$

where $d$ is the geodesic distance in $M$.

In the previous sections, we have asked that the metrics $h$ we consider on the manifold $N$ satisfy (8.6) and (8.7) or (9.3). This was needed to guarantee that the results we have obtained are independent of the choice of $h$, but might depend on $\Lambda$. We now restrict our attention to admissible hypersurfaces $N$ which are embedded in $M$ and which are close to some reference smooth admissible hypersurface $N_{0}$. To make things precise, we consider a smooth admissible hypersurfaces $N_{0}$ and assume that any admissible hypersurface $N$ we consider can be written as a geodesic normal graph over $N_{0}$ for some function $\psi_{N} \in \mathcal{C}^{\ell, \alpha}\left(N_{0}\right)$.

Definition 10.1. The $\mathcal{C}_{\varepsilon}^{\ell, \alpha}$ norm of the hypersurface $N$, with respect to the hypersurface $N_{0}$, is defined to be

$$
\|N\|_{\mathcal{C}_{\varepsilon}^{\ell, \alpha}\left(N_{0}\right)}:=\left\|\psi_{N}\right\|_{\mathcal{C}_{\varepsilon}^{\ell, \alpha}\left(N_{0}\right)},
$$

where

$$
\begin{aligned}
\|\psi\|_{\mathcal{C}_{\varepsilon}^{\ell, \alpha}\left(N_{0}\right)} & :=\|\psi\|_{L^{\infty}\left(N_{0}\right)}+\|\nabla \psi\|_{L^{\infty}\left(N_{0}\right)} \\
& +\sum_{j=2}^{\ell} \varepsilon^{j-2}\left\|\nabla^{j} \psi\right\|_{L^{\infty}\left(N_{0}\right)}+\varepsilon^{\ell-2+\alpha}\left\|\nabla^{\ell} \psi\right\|_{\mathcal{C}^{0, \alpha}\left(N_{0}\right)},
\end{aligned}
$$

and where $\psi_{N}$ is the function whose normal geodesic graph over $N_{0}$ is $N$.

Since the hypersurface $N$ is assumed to be embedded in $M$, the metric $h$ we consider on $N$ is just the metric induced by the metric $g$ of $M$. Now, given $\Lambda>0$ and $\ell \geqslant 2$, one can find $\Lambda^{\prime}>0$ such that : If the $\mathcal{C}_{\varepsilon}^{\ell, \alpha}\left(N_{0}\right)$ norm of $\psi_{N}$ is bounded by $\Lambda^{\prime}$, then conditions (8.6) and (9.3) are fulfilled and $N$ is embedded.

Remark 10.2. As in (9.4) the definition of the weighted norm of a hypersurface is not the natural one, namely

$$
|N|_{\mathcal{C}_{\varepsilon}^{\ell, \alpha}\left(N_{0}\right)}:=\left|\psi_{N}\right|_{\mathcal{C}_{\varepsilon}^{\ell, \alpha}\left(N_{0}\right)}
$$


where

$$
|\psi|_{\mathcal{C}_{\varepsilon}^{\ell, \alpha}\left(N_{0}\right)}:=\sum_{j=0}^{\ell} \varepsilon^{j}\left\|\nabla^{j} \psi\right\|_{L^{\infty}\left(N_{0}\right)}+\varepsilon^{\ell+\alpha}\left\|\nabla^{\ell} \psi\right\|_{\mathcal{C}^{0, \alpha}\left(N_{0}\right)} .
$$

In order to justify this choice, observe that, as already mentioned, Definition 10.1 is consistent with the definition of the norm of the induced metric which is used in $\S 9.2$, while the above definition is consistent with the definition used in Remark 9.3.

In order to define Fermi coordinates in some neighborhood of $N$ we set for all $t \in[-\tau, \tau]$ and all $y \in N$

$$
Z_{N}(t, y):=\exp _{y}\left(t \nu_{y}\right)
$$

where $\nu_{y}$ is the normal to $N$ at the point $y$, which is assumed to point towards $M^{+}(N)$. The parameter $\tau$ is chosen small enough so that $Z_{N}$ is a diffeomorphism onto its image.

Definition 10.3. The coordinates $(t, y)$, defined in $V_{\tau}(N)$, are called Fermi coordinates relative to the hypersurface $N$.

We will need the following classical result which states that the distance function to $N$ is well-defined and as smooth as $N$ in some tubular neighborhood of $N$ whose width is bounded from below. The proof of this result can be found in [10] or in [9].

Lemma 10.4. Assume that $\ell \geqslant 2$ and that $\Lambda^{\prime}>0$ is fixed small enough. Then, there exists $\tau_{0}>0$ only depending on $\Lambda^{\prime}$ such that if $N$ is a hypersurface whose $\mathcal{C}_{\varepsilon}^{\ell, \alpha}\left(N_{0}\right)$ norm is bounded by $\Lambda^{\prime}$, then $Z_{N}$ is a $\mathcal{C}^{\ell-1, \alpha}$ diffeomorphism from $\left(-\tau_{0}, \tau_{0}\right) \times N$ onto its image. Moreover the norm of $\nabla d_{N}$ in $\mathcal{C}_{\varepsilon}^{\ell-1, \alpha}\left(V_{\tau_{0}}(N)\right)$ is bounded by a constant which only depends on $\Lambda^{\prime}$.

Proof. The existence of the constant $\tau_{0}$ follows from the fact that the hypersurfaces we consider are uniformly bounded in $\mathcal{C}^{2}$ topology. This implies that the principal curvatures of $N$ are bounded from above by some constant only depending on $\Lambda^{\prime}$ and, in turn, this shows that the size of the tubular neighborhood over which $Z_{N}$ is a diffeomorphism is bounded from below.

The fact that $d_{N}$ is as regular as $N$ follows from standard arguments. Indeed, the gradient of $d_{N}$ at the point of coordinates $(t, y)$ is given by the tangent vector to the geodesic starting from $y$ with vector speed $\nu_{y}$. Hence the gradient of $d_{N}$ is as regular as the Gauss map of $N$, which in 
turn is as regular as $\nabla \psi_{N}$. Hence $\nabla d_{N}$ is bounded in $\mathcal{C}_{\varepsilon}^{\ell-1, \alpha}\left(V_{\tau_{0}}\right)$ by $\Lambda^{\prime}$ (we even know that $\nabla^{2} d_{N}$ is bounded in $\mathcal{C}_{\varepsilon}^{\ell-2, \alpha}\left(V_{\tau_{0}}\right)$ by some constant only depending on $\Lambda^{\prime}$, which is a slightly stronger statement). q.e.d.

Let us recall that

$$
\left|\nabla d_{N}\right|_{g}=1
$$

a.e. in $M$.

From now on, we assume that $\tau_{0}$ is chosen as in Lemma 10.4. For all $t \in\left[-\tau_{0}, \tau_{0}\right]$, we define the hypersurface $N_{t}$ to be the hypersurface which is parallel to $N$, at distance $t$. This means that $N_{t}$ is the normal geodesic graph over $N$ for the constant function $\psi(y) \equiv t$. This definition being understood, we recall the expression of the Laplace-Beltrami operator in Fermi coordinates.

Lemma 10.5. Denote by $(t, y)$ the Fermi coordinates relative to $N$, which are defined in $V_{\tau_{0}}(N)$. Then, at any point of $Z_{N}(t, y) \in N_{t}$ we have

$$
\Delta_{g}=\partial_{t}^{2}+\Delta_{h_{t}}-n H_{N_{t}} \partial_{t}
$$

where $h_{t}$ is the metric induced by $g$ on $N_{t}$ and where $H_{N_{t}}(t, y)$ denotes the mean curvature of the hypersurface $N_{t}$ at the point $Z_{N}(t, y)$.

Proof. We provide the proof of the formula when $t=0$. The general case can be treated similarly, up to notation changes. Let $e_{1}, \ldots, e_{n}$ be an orthonormal frame field on $N$ and $\nu$ be the normal vector field.

The Laplace-Beltrami operator on $M$ is defined by

$$
\Delta_{g}=\sum_{i=1}^{n}\left(e_{i} e_{i}-D_{e_{i}} e_{i}\right)+\nu \nu-D_{\nu} \nu
$$

where $D$ is the Levi-Civita connection on $M$. Let $D^{N}$ denote the LeviCivita connection on $N$, by construction, we have

$$
D_{e_{i}} e_{i}=D_{e_{i}}^{N} e_{i}+g\left(D_{e_{i}} e_{i}, \nu\right) \nu
$$

Therefore

$$
\Delta_{g}=\sum_{i=1}^{n}\left(e_{i} e_{i}-D_{e_{i}}^{N} e_{i}\right)+\sum_{i=1}^{n} g\left(e_{i}, D_{e_{i}} \nu\right) \nu+\nu \nu-D_{\nu} \nu .
$$


By definition $\nu \nu=\partial_{t}^{2}$ and $\nu=\partial_{t}$. Furthermore $D_{\nu} \nu=0$ and

$$
\sum_{i=1}^{n} g\left(e_{i}, D_{e_{i}} \nu\right)=-n H_{N},
$$

where $H_{N}$ is the mean curvature of $N$. Hence we conclude that

$$
\Delta_{g}=\partial_{t}^{2}+\Delta_{h}-n H_{N} \partial_{t}
$$

Which is the desired expression.

q.e.d.

Applying the expansion given in the previous Lemma to the function $d_{N}(t, y)=t$, we obtain the important formula

$$
\Delta_{g} d_{N}(t, y)=-n H_{N_{t}}(t, y)
$$

When $t=0$ we recover the well-known fact that the Laplacian of the distance function to the hypersurface $N$, computed at a point of $N$, is equal to (minus) the sum of the principal curvatures of the hypersurface at this point [9].

We will also need the expansion of the Laplace-Beltrami operator $\Delta_{g}$ in Fermi coordinates. To do so, we need slightly better control on the regularity of the hypersurfaces $N$ which should be at least bounded in $\mathcal{C}_{\varepsilon}^{3, \alpha}\left(N_{0}\right)$.

Proposition 10.6. Assume that $\Lambda^{\prime}>0$ is fixed small enough. If $N$ is a hypersurface whose $\mathcal{C}_{\varepsilon}^{3, \alpha}\left(N_{0}\right)$ norm is bounded by $\Lambda^{\prime}$, then there exists a second order operator in $\partial_{z_{j}}$

$$
L_{2}:=t \mathcal{O}_{\mathcal{C}_{\varepsilon}^{1, \alpha}}\left(\Lambda^{\prime}\right) \nabla^{2},
$$

without any first or zero-th order term, and a first order operator in $\partial_{t}$ and $\partial_{z_{j}}$

$$
L_{1}:=\mathcal{O}_{\mathcal{C}_{\varepsilon}^{0, \alpha}}\left(\Lambda^{\prime}\right) \nabla+t \varepsilon^{-1} \mathcal{O}_{\mathcal{C}_{\varepsilon}^{0, \alpha}}\left(\Lambda^{\prime}\right) \nabla
$$

without zero-th order term, such that

$$
\Delta_{g}=\partial_{t}^{2}+\Delta_{h}+L_{1}+L_{2}
$$

where $h$ is the metric on $N$, induced by the metric $g$ on $M$. The notation $\mathcal{O}_{\mathcal{C}_{\varepsilon}^{k, \alpha}}\left(\Lambda^{\prime}\right)$ refers to the fact that the coefficients of the operators are bounded in $\mathcal{C}_{\varepsilon}^{k, \alpha}\left(V_{\tau_{0}}(N)\right)$ by a constant which only depends on $\Lambda^{\prime}$. 
Proof. This result follows from the expansion of the metric in Fermi coordinates $(t, y)$ relative to $N$. Indeed, if we write the metric $g$ as

$$
g=g_{t t} d t \otimes d t+\sum_{j=1}^{n} g_{t j} d t \otimes d z_{j}+\sum_{j, j^{\prime}=1}^{n} g_{j j^{\prime}} d z_{j} \otimes d z_{j^{\prime}}
$$

the coefficients of the metric can be expanded as

$$
g_{t t}=1, \quad g_{t j}=0 \quad \text { and } \quad g_{i j}=h_{i j}+t \mathcal{O}_{\mathcal{C}_{\varepsilon}^{\ell-1, \alpha}}\left(\Lambda^{\prime}\right),
$$

where $h_{i j}$ are the coefficients of the induced metric on $N$. This is precisely at this point that we use the definition (10.2). Indeed, the regularity of the coefficients of the metric $g$ in Fermi coordinates is the same as the regularity of the gradient of the Gauss map of $N$, which in turn can be estimated by the second derivatives of the function whose graph is $N$. Hence, the $\mathcal{C}_{\varepsilon}^{1, \alpha}\left(V_{\tau_{0}}(N)\right)$ norm of the coefficients of the metric is bounded by the $\mathcal{C}_{\varepsilon}^{\ell+1, \alpha}\left(N_{0}\right)$ norm of $N$.

Similar expansions hold for $g^{\alpha \beta}$, the coefficients of $g^{-1}$. This implies that

$$
\sqrt{\operatorname{det} g}=\sqrt{\operatorname{det} h}+t \mathcal{O}_{\mathcal{C}_{\varepsilon}^{1, \alpha}}\left(\Lambda^{\prime}\right)
$$

With these expansions, we get

$$
\frac{1}{\sqrt{\operatorname{det} g}} \partial_{t}\left(\sqrt{\operatorname{det} g} g^{t t} \partial_{t}\right)=\partial_{t}^{2}+\left(\mathcal{O}_{\mathcal{C}_{\varepsilon}^{1, \alpha}}\left(\Lambda^{\prime}\right)+t \varepsilon^{-1} \mathcal{O}_{\mathcal{C}_{\varepsilon}^{0, \alpha}}\left(\Lambda^{\prime}\right)\right) \partial_{t}
$$

and

$$
\begin{aligned}
\frac{1}{\sqrt{\operatorname{det} g}} \partial_{z_{i}}\left(\sqrt{\operatorname{det} g} g^{i j} \partial_{z_{j}}\right)= & \frac{1}{\sqrt{\operatorname{det} h}} \partial_{z_{i}}\left(\sqrt{\operatorname{deth}} h^{i j} \partial_{z_{j}}\right) \\
& +t \varepsilon^{-1} \mathcal{O}_{\mathcal{C}_{\varepsilon}^{0, \alpha}}\left(\Lambda^{\prime}\right) \partial_{z_{j}}+t \mathcal{O}_{\mathcal{C}_{\varepsilon}^{1, \alpha}}\left(\Lambda^{\prime}\right) \partial_{z_{i}} \partial_{z_{j}}
\end{aligned}
$$

The result now follows at once.

q.e.d.

As promised, we now explain the modifications needed when $\partial N \neq$ $\emptyset$. Observe that, when $\partial N=\emptyset$, the geodesics starting from a point $y \in N$ with initial velocity $\nu_{N}(y)$ induce a fibration of a tubular neighborhood of $N$. In the case where $N$ has a boundary this property is not true anymore. To overcome this problem, we define what we call "twisted" Fermi coordinates. Recall that we have assumed in the introduction that $M$ is a smooth domain of a Riemannian manifold $\widetilde{M}$. An 
admissible hypersurface $N$ in $M$ can be extended to a hypersurface $\widetilde{N}$ whose $\mathcal{C}_{\varepsilon}^{\ell, \alpha}$ norm is bounded by a constant (independent of $\varepsilon$ ) times the $\mathcal{C}_{\varepsilon}^{2, \alpha}$ norm of $N$.

Observe that, provided $\tau_{0}$ is chosen small enough, we can define Fermi coordinates in

$$
V_{\tau_{0}}(\tilde{N}):=\left\{Z_{\widetilde{N}}(t, y) \in \widetilde{M}: y \in \widetilde{N}, \quad t \in\left(-\tau_{0}, \tau_{0}\right)\right\} .
$$

Let $X_{1}$ be the vector field $\partial_{t}$, which is defined in $V_{\tau_{0}}(\tilde{N})$. Reducing $\tau_{0}$ if this is necessary, we can assume that $\nu_{\partial M}$, the inner normal to $\partial M$, does not coincide with $X_{1}$ in $V_{\tau_{0}}(\widetilde{N})$ (recall that $N$ is assumed to meet $\partial M$ in an orthogonal way).

We set $X_{2}$ to be the extension of $\nu_{\partial M}$ which is given by the unit normal vector fields to the family of parallel hypersurfaces to $\partial M$. This vector field is well-defined in a fixed tubular neighborhood of radius $\varepsilon_{0}>0$ of $\partial M$. Finally, we consider a smooth cut-off function $\eta$ which is identically equal to 1 in $\left(-\infty, \varepsilon_{0} / 2\right)$ and equal to 0 in $\left(\varepsilon_{0}, \infty\right)$, and let

$$
\tilde{\chi}:=\eta\left(d_{\partial M}\right),
$$

where $d_{\partial M}$ is the distance function to $\partial M$. We define the vector field

$$
X:=\frac{X_{1}-\left\langle X_{1}, \tilde{\chi} X_{2}\right\rangle_{g} \tilde{\chi} X_{2}}{1-\left\langle X_{1}, \tilde{\chi} X_{2}\right\rangle_{g}^{2}} .
$$

It is straightforward to check the following properties of $X$ :

(i) $X$ is tangent to $\partial M$.

(ii) $X=X_{1}$ at a point $p$ whenever $d_{\partial M}(p) \geqslant \varepsilon_{0}$.

(iii) $\left\langle X, X_{1}\right\rangle_{g} \equiv 1$.

We now define $\left\{F_{t}\right\}_{t}$ to be the flow associated to $X$. It follows from (i) that $F_{t}(N)$ is contained in $M$. Also, (i) and (iii) imply that $(t, y) \rightarrow$ $F_{t}(y)$ is in fact a diffeomorphism from $\left(-\tau_{0}, \tau_{0}\right) \times N$ onto $V_{\tau_{0}}(\widetilde{N}) \cap M$.

The "twisted" Fermi coordinates $(t, y)$ relative to $N$ are defined by:

$$
Y_{N}(t, y):=F_{t}(y)
$$

Observe that (ii) implies that $Y_{N}(t, y)=Z_{N}(t, y)$ away from a tubular neighborhood of radius $\varepsilon_{0}$ around $\partial M$. Moreover, (iii) together with the 
fact that, $N$ being an admissible hypersurface, $N$ meets $\partial M$ orthogonally, imply that in a neighborhood of radius $\varepsilon_{0}$ of $\partial M$, if $(t, y)$ are the "twisted" Fermi coordinates of a point $p$ (relative to $N$ ) and if $(\widetilde{t}, \widetilde{y})$ are the Fermi coordinates of the same point $p$ (relative to $\widetilde{N}$ ), we have

$$
t=\tilde{t}, \quad \text { and } \quad d(\widetilde{y}, y)=\mathcal{O}\left(t^{2}\right) .
$$

Using these properties, one checks that all the results we have obtained in this section do hold when Fermi coordinates are replaced by "twisted" Fermi coordinates.

One last comment about the modifications which are needed when $\partial N$ is not empty. In the case where $\partial N$ is empty, one can parameterize any hypersurface $\widetilde{N}$ close enough to $N$ as a normal geodesic graph, that is, using the flow $Z_{N}$. In turn, when $\partial N$ is not empty, we agree that we will parameterize any hypersurface $\widetilde{N}$ close enough to $N$ using the flow

$Y_{N}$. With slight abuse of terminology we will say that $\widetilde{N}$ is a "normal graph" over $N$.

\section{The linear problem}

Assume that $(M, g)$ is a smooth $(n+1)$-dimensional Riemannian manifold, with or without boundary and that $N_{0} \subset M$ is a fixed admissible, nondegenerate minimal hypersurface or a volume-nondegenerate constant mean curvature hypersurface.

\subsection{Preliminary assumptions and definitions}

From now on, we assume that $\ell \geqslant 2$ and that $\Lambda^{\prime}>0$ is fixed small enough. We also agree that any admissible hypersurface $N$ we consider satisfies:

$\left(H\left(\ell, \Lambda^{\prime}\right)\right) \quad$ The $\mathcal{C}_{\varepsilon}^{\ell, \alpha}\left(N_{0}\right)$ norm of the function $\psi_{N}$, whose normal
graph is the hypersurface $N$, is bounded by $\Lambda^{\prime}$.

We assume that $\Lambda^{\prime}$ is chosen so that whenever $N$ satisfies the above conditions, then $N$ is embedded and (8.6) and (9.3) are fulfilled. Furthermore, reducing $\Lambda^{\prime}$ if this is necessary, we can assume that $N \subset$ $V_{\tau_{0} / 4}\left(N_{0}\right)$ where $\tau_{0}$ is chosen as in Lemma 10.4.

We choose a cutoff function $\chi$ which is identically equal to 1 in $\left(-\tau_{0} / 2, \tau_{0} / 2\right)$ and identically equal to 0 outside $\left[-\tau_{0}, \tau_{0}\right]$. We define

$$
u_{\varepsilon, N}:=\chi\left(d_{N}\right) \frac{d_{N}}{\left|d_{N}\right|}+\left(1-\chi\left(d_{N}\right)\right) u_{\star}\left(d_{N} / \varepsilon\right) .
$$




\subsection{Function spaces}

Paralleling what we have done in $\S 9$, we define weighted Hölder spaces adapted to our problem. We first give the definition of the Hölder spaces which take into account the scaling parameter $\varepsilon$.

Definition 11.1. Given $\ell \in \mathbb{N}, \alpha \in(0,1)$ and $\varepsilon \in(0,1)$, we define the space $\mathcal{C}_{\varepsilon}^{\ell, \alpha}(M)$ to be the space of functions which are $\ell$ times differentiable and whose $\ell$-th partial derivatives are Hölder of exponent $\alpha$. This space is endowed with the norm

$$
\|u\|_{\mathcal{C}_{\varepsilon}^{\ell, \alpha}(M)}:=\sum_{j=0}^{\ell^{\prime}} \varepsilon^{j}\left\|\nabla^{j} u\right\|_{L^{\infty}(M)}+\varepsilon^{\ell+\alpha} \sup _{p \neq q \in M} \frac{\left|\nabla^{\ell} u(p)-\nabla^{\ell} u(q)\right|}{d(p, q)^{\alpha}}
$$

where $d$ is the geodesic distance in $M$.

This definition being understood, we define a projection operator:

Definition 11.2. Denote by $(t, y)$ the (twisted) Fermi coordinates relative to $N$. For all $u \in \mathcal{C}^{\ell, \alpha}(M)$, we define the function $\Pi_{\varepsilon, N}(u)$ by

$$
\Pi_{\varepsilon, N}(u)(t, y):=u(t, y)-\frac{\int_{\mathbb{R}} \chi(s) u(s, y) w_{\star}(s / \varepsilon) d s}{\int_{\mathbb{R}} \chi^{2}(s) w_{\star}^{2}(s / \varepsilon) d s} \chi(t) w_{\star}(t / \varepsilon),
$$

in $V_{\tau_{0}}(N)$ and $\Pi_{\varepsilon, N}(u)=u$ in $M-V_{\tau_{0}}(N)$.

Observe that $\Pi_{\varepsilon, N}$ is an involution since, by construction $\Pi_{\varepsilon, N} \circ$ $\Pi_{\varepsilon, N}=\Pi_{\varepsilon, N}$. It is straightforward to check the:

Lemma 11.3. Assume that $\left(H\left(3, \Lambda^{\prime}\right)\right)$ holds. Then, for all $\ell=$ $0, \ldots, 2$

$$
\Pi_{\varepsilon, N}: \mathcal{C}_{\varepsilon}^{\ell, \alpha}(M) \longrightarrow \mathcal{C}_{\varepsilon}^{\ell, \alpha}(M),
$$

is well-defined and uniformly bounded for $\varepsilon \in(0,1)$.

Proof. The fact that this operator is well-defined follows from the fact that Fermi coordinates induce a local $\mathcal{C}^{2, \alpha}$ diffeomorphism $\mathcal{D}$ when $N$ is a $\mathcal{C}^{3, \alpha}$ hypersurface.

The fact that $\Pi_{\varepsilon, N}$ is bounded uniformly between the weighted spaces follows at once from the definition of the $\mathcal{C}_{\varepsilon}^{3, \alpha}\left(N_{0}\right)$ norm of $N$ which ensures that the diffeomorphism $\mathcal{D}$ is uniformly bounded 
in $L^{\infty}\left(V_{\tau_{0}}(N)\right)$ and has a differential which is uniformly bounded in $\mathcal{C}_{\varepsilon}^{1}\left(V_{\tau_{0}}(N)\right)$.

q.e.d.

We now define weighted function spaces on $M$ which parallel the spaces $\mathcal{E}_{\delta, \varepsilon}^{\ell, \alpha}(\mathbb{R} \times N)$, when the weights $\delta_{ \pm}$are chosen to be equal to 0 .

Definition 11.4. Assume that $\left(H\left(\ell+1, \Lambda^{\prime}\right)\right)$ holds for some $\ell \geqslant 2$. Given $0 \leqslant \ell^{\prime} \leqslant \ell, \alpha \in(0,1)$ and $\varepsilon \in(0,1)$, we define the space $\mathcal{E}_{\varepsilon, N}^{\ell^{\prime}, \alpha}(M)$ to be the space of functions $u \in \mathcal{C}_{\varepsilon}^{\ell^{\prime}}, \alpha(M)$ which satisfy

$$
\Pi_{\varepsilon, N}(u)=u .
$$

This space is endowed with the induced norm.

As usual, we also define $\left[\mathcal{C}_{\varepsilon}^{\ell^{\prime}, \alpha}(M)\right]_{0}\left(\right.$ resp. $\left.\left[\mathcal{E}_{\varepsilon, N}^{\ell^{\prime}, \alpha}(M)\right]_{0}\right)$ to be the subspace of functions of $\mathcal{C}_{\varepsilon}^{\ell^{\prime}, \alpha}(M)$ (resp. $\mathcal{E}_{\varepsilon, N}^{\ell^{\prime}, \alpha}(M)$ ) which have 0 Neumann boundary condition on $\partial M$, if this boundary is not empty.

\subsection{The linear problem}

Assume that $N$ is an admissible hypersurface which satisfies $\left(H\left(2, \Lambda^{\prime}\right)\right)$. Let $u_{\varepsilon, N}$ be defined as in (11.1), the linearized operator we are interested in reads

$$
\mathbb{L}_{\varepsilon, N}:=-\varepsilon^{2} \Delta_{g}+\frac{1}{2} W^{\prime \prime}\left(u_{\varepsilon, N}\right) .
$$

In this section, we would like to construct a right inverse for this operator. To do so, we first define and study the auxiliary operator

$$
\mathbb{L}_{0}:=-\varepsilon^{2} \Delta_{g}+\frac{1}{2} \Gamma
$$

where the potential $\Gamma$ is chosen to interpolate smoothly between $2 \gamma_{-}^{2}=$ $W^{\prime \prime}(-1)$ in $M^{-}(N)$ and $2 \gamma_{+}^{2}=W^{\prime \prime}(1)$ in $M^{+}(N)$. More precisely, let $\xi$ denote a smooth cutoff function equal to 1 on $(1,+\infty)$ and equal to 0 on $(-\infty,-1)$, with $\xi \geqslant 0$. If $(t, y)$ are (twisted) Fermi coordinates relative to $N$, we define

$$
\Gamma(t, y):=2\left((1-\xi(t / \varepsilon)) \gamma_{-}^{2}+\xi(t / \varepsilon) \gamma_{+}^{2}\right),
$$

in $V_{\tau_{0}}(N)$ and

$$
\Gamma:=2 \gamma_{ \pm}^{2}
$$

in $M^{ \pm}(N)-V_{\tau_{0}}(N)$. We have: 
Lemma 11.5. There exists $\varepsilon_{0}>0$ only depending on $\Lambda^{\prime}$ such that, for all $\varepsilon \in\left(0, \varepsilon_{0}\right)$, the operator

$$
\mathbb{L}_{0}:\left[\mathcal{C}_{\varepsilon}^{0, \alpha}(M)\right]_{0} \longrightarrow \mathcal{C}_{\varepsilon}^{2, \alpha}(M),
$$

is an isomorphism the norm of whose inverse is bounded by some constant which depends on $\Lambda^{\prime}$ but does not depend on $\varepsilon$ nor on $N$ satisfying $\left(H\left(2, \Lambda^{\prime}\right)\right)$.

Proof. The fact that $\mathbb{L}_{0}$ is an isomorphism, clearly follows from the fact that the potential $\Gamma$ is bounded from below by a positive constant independent of $\varepsilon$. In particular, the constant function 1 can be used as a barrier to show that

$$
\|w\|_{L^{\infty}(M)} \leqslant c\left\|\mathbb{L}_{0} w\right\|_{L^{\infty}(M)},
$$

for some constant $c>0$ which does not depend on $\varepsilon$. Then, the estimates for the derivatives of $w$ are consequences of Schauder's estimates on geodesic balls of radius $\varepsilon$. $\quad$ q.e.d.

Collecting the results of the previous sections we construct for the operator $\mathbb{L}_{\varepsilon, N}$ a right inverse whose norm is uniformly bounded as $\varepsilon$ tends to 0 . The construction of the right inverse relies on all our former analysis. To be more precise, we glue together local parametrizes given by Proposition 9.4 and Lemma 11.5 and obtain the right inverse for $\mathbb{L}_{\varepsilon, N}$ by applying a perturbation argument together with Proposition 10.6. As will become clear in the proof and in the statement of the next result, we need to assume that the submanifold $N$ has one degree of regularity higher than would be expected. Indeed, a natural guess would be that, in order to solve the equation $\mathbb{L}_{\varepsilon, N} w=f \in \mathcal{C}^{k-2, \alpha}$ in some Hölder space $\mathcal{C}^{k, \alpha}$, one would need to assume that the hypersurface $N$ is itself $\mathcal{C}^{k, \alpha}$. However, our construction relies on the use of Proposition 10.6 together with the use of the projection operator $\Pi_{\varepsilon, N}$ which both require $N$ to be a $\mathcal{C}^{k+1, \alpha}$ hypersurface. We now state the main technical result of our paper:

Proposition 11.6. Assume $N$ satisfies $\left(H\left(3, \Lambda^{\prime}\right)\right)$. Then, there exists $\varepsilon_{0}>0$ only depending on $\Lambda^{\prime}$ such that, for all $\varepsilon \in\left(0, \varepsilon_{0}\right)$, there exists an operator

$$
\mathbb{G}_{\varepsilon, N}: \mathcal{E}_{\varepsilon, N}^{0, \alpha}(M) \longrightarrow\left[\mathcal{E}_{\varepsilon, N}^{2, \alpha}(M)\right]_{0},
$$

satisfying

$$
\Pi_{\varepsilon, N} \circ \mathbb{L}_{\varepsilon, N} \circ \mathbb{G}_{\varepsilon, N}=I .
$$


Furthermore the norm of $\mathbb{G}_{\varepsilon, N}$ is bounded by some constant which depends on $\Lambda^{\prime}$, but does not depend on $\varepsilon \in\left(0, \varepsilon_{0}\right)$ nor on $N$.

Proof. The construction of $\mathbb{G}_{\varepsilon, N}$ is decomposed in 4 steps. As already mentioned we "glue" together different parametrizes which have been defined in the previous sections. In the first step, we use the result of Lemma 11.5 to reduce the solvability of $\Pi_{\varepsilon, N} \circ \mathbb{L}_{\varepsilon, N} w=f$ to the case where $f$ is supported in $V_{\tau_{0}}(N)$. This allows us to use, in Step 2 the result of Proposition 9.4 where a right inverse for the operator $\mathfrak{L}_{\varepsilon}$ has been constructed. In Step 3, we use the expansion of the Laplace-Beltrami operator in Fermi coordinates which is provided by Proposition 10.6 to estimate the difference between the operators $\mathbb{L}_{\varepsilon, N}$ and the operator $\mathfrak{L}_{\varepsilon}$. At this point we have produced a bounded operator $G$ which is almost a right inverse. In Step 4, we apply a standard perturbation argument to find a right inverse for $\Pi_{\varepsilon, N} \circ \mathbb{L}_{\varepsilon, N}$.

In the proof, the constant $c>0$, which may vary from line to line, may depend on $\Lambda^{\prime}$, but does not depend on $\varepsilon$ (provided this parameter is chosen small enough), does not depend on $f$, nor on $N$ chosen to satisfy $\left(H\left(3, \Lambda^{\prime}\right)\right)$.

Step 1. Thanks to the result of Lemma 11.5, we find $w_{1} \in\left[\mathcal{C}_{\varepsilon}^{2, \alpha}(M)\right]_{0}$ solution of $\mathbb{L}_{0} w_{1}=f$. Furthermore, we know that

$$
\left\|w_{1}\right\|_{\mathcal{C}_{\varepsilon}^{2, \alpha}(M)} \leqslant c\|f\|_{\mathcal{C}_{\varepsilon}^{0, \alpha}(M)} .
$$

Now, the result of Lemma 11.3 implies that we also have

$$
\left\|\Pi_{\varepsilon, N} w_{1}\right\|_{\mathcal{C}_{\varepsilon}^{2, \alpha}(M)} \leqslant c\|f\|_{\mathcal{C}_{\varepsilon}^{0, \alpha}(M)} \cdot
$$

Observe that, as mentioned, we already need to assume that the hypersurface $N$ has bounded norm in $\mathcal{C}_{\varepsilon}^{3, \alpha}\left(N_{0}\right)$.

We define the function

$$
g:=f-\mathbb{L}_{\varepsilon, N} \circ \Pi_{\varepsilon, N} w_{1} .
$$

Since, away from $V_{\tau_{0}}(N)$, we have $\Pi_{\varepsilon, N} u=u$ and $\mathbb{L}_{0}=\mathbb{L}_{\varepsilon, N}$, we conclude that, the function $g$ is supported in $V_{\tau_{0}}(N)$. If we identify $V_{\tau_{0}}(N)$ with $\left(-\tau_{0}, \tau_{0}\right) \times N$ via (twisted) Fermi coordinates, we can extend the function $g$ by 0 to all $\mathbb{R} \times N$. We claim that

$$
\|g\|_{\mathcal{C}_{-\gamma, \varepsilon}^{0, \alpha}(\mathbb{R} \times N)} \leqslant c\|f\|_{\mathcal{C}_{\varepsilon}^{0, \alpha}(M)},
$$


where $\gamma:=\left(\gamma_{-}, \gamma_{+}\right)$are the indicial roots defined in (6.2). Indeed, using (6.3) and (6.4), we see that $u_{\star}$ converges exponentially to \pm 1 at a rate which is dictated by the indicial roots $\gamma_{ \pm}$. This in turn shows that the difference between the potential of the operators $\mathbb{L}_{\varepsilon, N}$ and $\mathbb{L}_{0}$ converges exponentially to 0 at a rate which is dictated by the indicial roots $2 \gamma_{ \pm}$. Namely

$$
\left\|\Gamma-W^{\prime \prime}\left(u_{\star}(\cdot / \varepsilon)\right)\right\|_{\mathcal{C}_{-2 \gamma, \varepsilon}^{0, \alpha}(\mathbb{R} \times N)} \leqslant c .
$$

This inequality already implies that

$$
\left\|\mathbb{L}_{\varepsilon, N} w_{1}-f\right\|_{\mathcal{C}_{-2 \gamma, \varepsilon}^{0, \alpha}(\mathbb{R} \times N)} \leqslant c\|f\|_{\mathcal{C}_{\varepsilon}^{0, \alpha}(M)} .
$$

Furthermore, using the definition of $\prod_{\varepsilon, N}$ together with the fact that

$$
\left\|\chi w_{\star}(\cdot / \varepsilon)\right\|_{\mathcal{C}_{-\gamma, \varepsilon}^{2, \alpha}(\mathbb{R} \times N)} \leqslant c,
$$

we get

$$
\left\|\mathbb{L}_{\varepsilon, N}\left(w_{1}-\Pi_{\varepsilon, N} w_{1}\right)\right\|_{\mathcal{C}_{-\gamma, \varepsilon}^{0, \alpha}(\mathbb{R} \times N)} \leqslant c\|f\|_{\mathcal{C}_{\varepsilon}^{0, \alpha}(M)} .
$$

This completes the proof of the claim.

Step 2. We define the projection operator

$$
\Pi_{\varepsilon, N}^{0}(u)(t, y):=u(t, y)-\frac{\int_{\mathbb{R}} u(s, y) w_{\star}(s / \varepsilon) d s}{\int_{\mathbb{R}} w_{\star}^{2}(s / \varepsilon) d s} w_{\star}(t / \varepsilon) .
$$

Clearly,

$$
\Pi_{\varepsilon, N}^{0}: \mathcal{C}_{-\gamma, \varepsilon}^{0, \alpha}(\mathbb{R} \times N) \longrightarrow \mathcal{E}_{-\gamma, \varepsilon}^{0, \alpha}(\mathbb{R} \times N),
$$

is bounded uniformly in $\varepsilon$. Hence, using (11.4), we conclude that

$$
\left\|\Pi_{\varepsilon, N}^{0} g\right\|_{\mathcal{C}_{-\gamma, \varepsilon}^{0, \alpha}(\mathbb{R} \times N)} \leqslant c\|f\|_{\mathcal{C}_{\varepsilon}^{0, \alpha}(M)} .
$$

We use the result of Proposition 9.4, with some fixed weights $\delta:=$ $\left(\delta_{-}, \delta_{+}\right)$satisfying $-\gamma_{ \pm}<\delta_{ \pm}<0$, to define $w_{2} \in\left[\mathcal{E}_{\delta, \varepsilon}^{2, \alpha}(\mathbb{R} \times N)\right]_{0}$ solution of

$$
\mathfrak{L}_{\varepsilon} w_{2}=\Pi_{\varepsilon, N}^{0} g
$$


where we recall that

$$
\mathfrak{L}_{\varepsilon}:=-\varepsilon^{2}\left(\partial_{t}^{2}+\Delta_{N}\right)+\frac{1}{2} W^{\prime \prime}\left(u_{\star}(\cdot / \varepsilon)\right) .
$$

Since the inverse of $\mathfrak{L}_{\varepsilon}$ has been shown to be uniformly bounded, we get the estimate

$$
\left\|w_{2}\right\|_{\mathcal{C}_{\delta, \varepsilon}^{2, \alpha}(\mathbb{R} \times N)} \leqslant c\left\|\Pi_{\varepsilon, N}^{0} g\right\|_{\mathcal{C}_{\delta, \varepsilon}^{0, \alpha}(\mathbb{R} \times N)} \leqslant c\left\|\Pi_{\varepsilon, N}^{0} g\right\|_{\mathcal{C}_{-\gamma, \varepsilon}^{0, \alpha}(\mathbb{R} \times N)},
$$

which, together with (11.6) implies that

$$
\left\|w_{2}\right\|_{\mathcal{C}_{\delta, \varepsilon}^{2, \alpha}(\mathbb{R} \times N)} \leqslant c\|f\|_{\mathcal{C}_{\varepsilon}^{0, \alpha}(M)} .
$$

Step 3. We claim that

$$
\left\|\Pi_{\varepsilon, N}\left(\mathbb{L}_{\varepsilon, N} \circ \Pi_{\varepsilon, N}\left(\chi w_{2}\right)-g\right)\right\|_{\mathcal{C}_{\varepsilon}^{0, \alpha}(M)} \leqslant c \varepsilon\|f\|_{\mathcal{C}_{\varepsilon}^{0, \alpha}(M)} .
$$

Since $w_{2}$ and $g$ are exponentially decaying in terms of $|t| / \varepsilon$, we readily have

$$
\left\|(1-\chi) \Pi_{\varepsilon, N}\left(\mathbb{L}_{\varepsilon, N} \circ \Pi_{\varepsilon, N}\left(\chi w_{2}\right)-g\right)\right\|_{\mathcal{C}_{\varepsilon}^{0, \alpha}(M)} \leqslant c e^{-\kappa_{1} / \varepsilon}\|f\|_{\mathcal{C}_{\varepsilon}^{0, \alpha}(M)},
$$

for some fixed $\kappa_{1}>0$ only depending on $\gamma, \delta$ and $\tau_{0}$. Hence, identifying $V_{\tau_{0}}(N)$ with $\left[-\tau_{0}, \tau_{0}\right] \times N$ as above, we only need to show that

$$
\left\|\chi \Pi_{\varepsilon, N}\left(\mathbb{L}_{\varepsilon, N} \circ \Pi_{\varepsilon, N}\left(\chi w_{2}\right)-g\right)\right\|_{\mathcal{C}_{\varepsilon}^{0, \alpha}(\mathbb{R} \times N)} \leqslant c \varepsilon\|f\|_{\mathcal{C}_{\varepsilon}^{0, \alpha}(M)} .
$$

The proof of this inequality essentially follows from the result of Proposition 10.6 which gives the expansion of $\Delta_{g}$ in Fermi coordinates relative to $N$. Observe that, again, we need to assume that the hypersurface $N$ has bounded norm in $\mathcal{C}_{\varepsilon}^{3, \alpha}\left(N_{0}\right)$ in order to apply the result of Proposition 10.6 with the correct regularity.

To begin with we replace $\Pi_{\varepsilon, N}$ by $\Pi_{\varepsilon, N}^{0}$ in the left-hand side of (11.9). This introduce a discrepancy which we now estimate. We set

$$
D_{1}:=\Pi_{\varepsilon, N} \circ \mathbb{L}_{\varepsilon, N} \circ \Pi_{\varepsilon, N}\left(\chi w_{2}\right)-\Pi_{\varepsilon, N}^{0} \circ \mathbb{L}_{\varepsilon, N} \circ \Pi_{\varepsilon, N}^{0}\left(\chi w_{2}\right),
$$

and

$$
D_{2}:=\Pi_{\varepsilon, N}^{0} g-\Pi_{\varepsilon, N} g
$$


Using the fact that $w_{\star}(\cdot / \varepsilon)$ is exponentially decaying in terms of $|t| / \varepsilon$, we see that the same is true for $D_{1}$ and $D_{2}$, so

$$
\left\|\chi\left(D_{1}+D_{2}\right)\right\|_{\mathcal{C}_{\varepsilon}^{0, \alpha}(\mathbb{R} \times N)} \leqslant c e^{-\kappa_{2} / \varepsilon}\|f\|_{\mathcal{C}_{\varepsilon}^{0, \alpha}(M)},
$$

for some fixed positive $\kappa_{2}$ which only depends on $\gamma, \delta$ and $\tau_{0}$.

Having replaced $\Pi_{\varepsilon, N}$ by $\Pi_{\varepsilon, N}^{0}$, we now replace $\chi w_{2}$ by $w_{2}$ in (11.9). This introduces yet another discrepancy which we now estimate. We set

$$
D_{3}:=\Pi_{\varepsilon, N}^{0} \circ \mathbb{L}_{\varepsilon, N} \circ \Pi_{\varepsilon, N}^{0}\left((1-\chi) w_{2}\right) .
$$

Since $w_{2}$ is exponentially decaying in terms of $|t| / \varepsilon$, we also have

$$
\left\|\chi D_{3}\right\|_{\mathcal{C}_{\varepsilon}^{0, \alpha}(\mathbb{R} \times N)} \leqslant c e^{-\kappa_{3} / \varepsilon}\|f\|_{\mathcal{C}_{\varepsilon}^{0, \alpha}(M)},
$$

for some fixed positive $\kappa_{3}$ which only depends on $\gamma, \delta$ and $\tau_{0}$.

Having done the above modifications, we are left with the estimate of

$$
\begin{aligned}
D_{4} & :=\Pi_{\varepsilon, N}^{0} \circ \mathbb{L}_{\varepsilon, N} \circ \Pi_{\varepsilon, N}^{0} w_{2}-\Pi_{\varepsilon, N}^{0} g \\
& =\Pi_{\varepsilon, N}^{0} \circ \mathbb{L}_{\varepsilon, N} w_{2}-\mathfrak{L}_{\varepsilon} w_{2} \\
& =\Pi_{\varepsilon, N}^{0}\left(\mathbb{L}_{\varepsilon, N} w_{2}-\mathfrak{L}_{\varepsilon} w_{2}\right) .
\end{aligned}
$$

We have used the fact that $\Pi_{\varepsilon, N}^{0} w_{2}=w_{2}$ since $w_{2} \in \mathcal{E}_{\delta, \varepsilon}^{2, \alpha}(\mathbb{R} \times N)$ and $\mathfrak{L}_{\varepsilon} w_{2}=\Pi_{\varepsilon}^{0} g$ to obtain the first identity. In order to obtain the second identity, we have used the fact that $\mathfrak{L}_{\varepsilon} w_{2}=\Pi_{\varepsilon, N}^{0} \mathfrak{L}_{\varepsilon} w_{2}$, by construction of the inverse of $\mathfrak{L}_{\varepsilon}$. At this point, we refer to the result of Proposition 10.6 which gives the expansion of $\Delta_{g}$ in Fermi coordinates about $N$ to get

$$
\mathbb{L}_{\varepsilon, N}-\mathcal{L}_{\varepsilon}=L_{1}+L_{2},
$$

where the properties of the operators $L_{1}$ and $L_{2}$ are stated in Proposition 10.6. These properties, together with (11.7), imply that

$$
\left\|\chi D_{4}\right\|_{\mathcal{C}_{\varepsilon}^{0, \alpha}(\mathbb{R} \times N)} \leqslant c \varepsilon\|f\|_{\mathcal{C}_{\varepsilon}^{0, \alpha}(M)} .
$$

This completes the proof of the claim.

Step 4. Collecting (11.8) together with the definition of $g$, we conclude that

$$
\left\|\Pi_{\varepsilon, N} \circ \mathbb{L}_{\varepsilon, N} \circ \Pi_{\varepsilon, N}\left(w_{1}+\chi w_{2}\right)-f\right\|_{\mathcal{C}_{\varepsilon}^{0, \alpha}(M)} \leqslant c \varepsilon\|f\|_{\mathcal{C}_{\varepsilon}^{0, \alpha}(M)} .
$$


Now, collecting (11.3) and (11.7) together with Lemma 11.3), we obtain

$$
\left\|\Pi_{\varepsilon, N}\left(w_{1}+\chi w_{2}\right)\right\|_{\mathcal{C}_{\varepsilon}^{2, \alpha}(M)} \leqslant c\|f\|_{\mathcal{C}_{\varepsilon}^{0, \alpha}(M)} .
$$

At this point, the existence of $\mathbb{G}_{\varepsilon, N}$ easily follows from a classical perturbation argument.

q.e.d.

Our next proposition gives an estimate of the norm of the operator

$$
\left(I-\Pi_{\varepsilon, N}\right) \circ \mathbb{L}_{\varepsilon, N} \circ \mathbb{G}_{\varepsilon, N},
$$

which, in some sense, measures the distance between the operator $\mathbb{L}_{\varepsilon, N} \circ$ $\mathbb{G}_{\varepsilon, N}$ and the identity in $\mathcal{E}_{\varepsilon}^{\ell-2, \alpha}(M)$. We define the operator $S_{\varepsilon, N}$ by

$$
S_{\varepsilon, N}(u)(y):=\int_{\mathbb{R}} \chi(s) u(t, y) w_{\star}(t / \varepsilon) d t,
$$

where $(t, y)$ are (twisted) Fermi coordinates relative to $N$ and $u$ is a function defined in $M$. Since $N$ is assumed to be a normal graph over $N_{0}$, any function on $N$ can be identified with a function on $N_{0}$ and its norm can be evaluated using the norms defined in (10.2) or in (10.3), provided $N$ is regular enough. This being understood, we have:

Proposition 11.7. Assume $N$ satisfies $\left(H\left(3, \Lambda^{\prime}\right)\right)$. Then, there exists $c>0$ only depending on $\Lambda^{\prime}$ such that, for all $w \in\left[\mathcal{E}_{\varepsilon, N}^{2, \alpha}(M)\right]_{0}$

$$
\left|S_{\varepsilon, N} \circ \mathbb{L}_{\varepsilon, N} w\right|_{\mathcal{C}_{\varepsilon}^{0, \alpha}\left(N_{0}\right)} \leqslant c \varepsilon^{2}\|w\|_{\mathcal{C}_{\varepsilon}^{2, \alpha}(M)}
$$

where the norm $|\cdot|_{\mathcal{C}_{\varepsilon}^{\ell, \alpha}\left(N_{0}\right)}$ is the one defined in (10.3).

Proof. We have to estimate

$$
y \longrightarrow \int_{\mathbb{R}} \chi(t) w_{\star}(t / \varepsilon) L_{\varepsilon, N} w(t, y) d t .
$$

We set

$$
E_{1}:=\int_{\mathbb{R}} \chi(t) w_{\star}(t / \varepsilon)\left(L_{\varepsilon, N}-\mathfrak{L}_{\varepsilon}\right) w(t, y) d t,
$$

where $\mathfrak{L}_{\varepsilon}$ is defined as in Step 2 of the previous proof. Using once more the result of Proposition 10.6, we obtain as in Step 3 of the previous proof

$$
\left|E_{1}\right|_{\mathcal{C}_{\varepsilon}^{0, \alpha}\left(N_{0}\right)} \leqslant c \varepsilon^{2}\|w\|_{\mathcal{C}_{\varepsilon}^{2, \alpha}(M)} .
$$


We set

$$
E_{2}:=\int_{\mathbb{R}} w_{\star}(t / \varepsilon)\left[\chi, \mathfrak{L}_{\varepsilon}\right] w(t, y) d t
$$

where $[A, B]$ is the commutator of the operators $A$ and $B$. Using the fact that $w_{\star}$ decays exponentially fast at $\pm \infty$, we conclude that $E_{2}$ decays exponentially fast in terms of $|t| / \varepsilon$. In particular

$$
\left|E_{2}\right|_{\mathcal{C}_{\varepsilon}^{0, \alpha}\left(N_{0}\right)} \leqslant c e^{-\kappa_{4} / \varepsilon}\|w\|_{\mathcal{C}_{\varepsilon}^{2, \alpha}(M)},
$$

for some fixed $\kappa_{4}>0$ which only depends on $\gamma$ and $\tau_{0}$.

Having estimated $E_{1}$ and $E_{2}$, it remains to estimate

$$
y \longrightarrow \int_{\mathbb{R}} w_{\star}(t / \varepsilon) \mathfrak{L}_{\varepsilon}(\chi w)(t, y) d t .
$$

We set

$$
E_{3}:=\int_{\mathbb{R}} w_{\star}(t / \varepsilon) \mathfrak{L}_{\varepsilon} \circ\left(\Pi_{\varepsilon, N}-\Pi_{\varepsilon, N}^{0}\right)(\chi w)(t, y) d t
$$

and

$$
E_{4}:=\int_{\mathbb{R}} w_{\star}(t / \varepsilon) \mathfrak{L}_{\varepsilon} \circ\left(I-\Pi_{\varepsilon, N}\right)(\chi w)(t, y) d t .
$$

Using the fact that $w_{\star}(\cdot / \varepsilon)$ is exponentially decaying in terms of $|t| / \varepsilon$, we get

$$
\left|E_{3}\right|_{\mathcal{C}_{\varepsilon}^{0, \alpha}\left(N_{0}\right)} \leqslant c e^{-\kappa_{5} / \varepsilon}\|u\|_{\mathcal{C}_{\varepsilon}^{2, \alpha}(M)},
$$

and, using in addition the fact that $\left(I-\Pi_{\varepsilon, N}\right) w=0$, we also have

$$
\left|E_{4}\right|_{\mathcal{C}_{\varepsilon}^{0, \alpha}\left(N_{0}\right)} \leqslant c e^{-\kappa_{5} / \varepsilon}\|u\|_{\mathcal{C}_{\varepsilon}^{2, \alpha}(M)},
$$

for some fixed $\kappa_{5}>0$ which only depends on $\gamma$ and $\tau_{0}$.

Having estimated $E_{1}$ through $E_{4}$, it finally remains to estimate

$$
y \longrightarrow \int_{\mathbb{R}} w_{\star}(t / \varepsilon) \mathfrak{L}_{\varepsilon} \circ \Pi_{\varepsilon, N}^{0}(\chi u)(t, y) d t .
$$

But, as we have already done in (9.7), one checks that this quantity is identically equal to 0 . This completes the proof of (11.11). q.e.d.

We would like to obtain similar results when $N$ is less regular than what is required in the last two results. To do so we introduce smoothing 
operators which will alow us to find $N_{\star}$, smooth enough so that we can apply the previous results, and close enough to $N$ so that a perturbation argument can be applied.

We recall from [1], page 97, that there exists a one parameter family of smoothing operators $\left(R_{\theta}\right)_{\theta \geqslant 1}$ and $C>0$ such that

$$
\begin{array}{rlrl}
\left\|R_{\theta} u\right\|_{\mathcal{C}^{k, \alpha}} & \leqslant C\|u\|_{\mathcal{C}^{k^{\prime}, \alpha^{\prime}}} & \text { for } k+\alpha \leqslant k^{\prime}+\alpha^{\prime} \\
\left\|R_{\theta} u\right\|_{\mathcal{C}^{k, \alpha}} & \leqslant C \theta^{k+\alpha-k^{\prime}-\alpha^{\prime}}\|u\|_{\mathcal{C}^{k^{\prime}, \alpha^{\prime}}} & \text { for } k+\alpha \geqslant k^{\prime}+\alpha^{\prime} \\
\left\|u-R_{\theta} u\right\|_{\mathcal{C}^{k, \alpha}} & \leqslant C \theta^{k+\alpha-k^{\prime}-\alpha^{\prime}}\|u\|_{\mathcal{C}^{k^{\prime}, \alpha^{\prime}}} & \text { for } & k+\alpha \leqslant k^{\prime}+\alpha^{\prime} .
\end{array}
$$

These operators act on functions defined in $\mathbb{R}^{n}$ but they can be localized and extended to functions defined on smooth manifolds using a partition of unity. We use $R_{\theta}$ to improve the regularity of a given hypersurface $N$ which is assumed to be a normal graph over $N_{0}$. We further assume that $\left(H\left(\ell, \Lambda^{\prime \prime}\right)\right)$ is satisfied with $\Lambda^{\prime \prime}$ smaller than the usual constant $\Lambda^{\prime}$. Hence, $N$ is the normal graph over $N_{0}$ for some function $\psi$ which satisfies

$$
\|\psi\|_{\mathcal{C}_{\varepsilon}^{\ell, \alpha}\left(N_{0}\right)} \leqslant \Lambda^{\prime \prime}
$$

for some $\ell \geqslant 2$. We define the function $\psi_{\star}:=R_{1 / \varepsilon} \psi$, and we define the hypersurface $N_{\star}$ to be the graph of the function $\psi_{\star}$ over $N_{0}$. We will write

$$
N_{\star}=R_{1 / \varepsilon} N
$$

We claim that,

$$
\left|\psi_{\star}-\psi\right|_{\mathcal{C}_{\varepsilon}^{\ell, \alpha}\left(N_{0}\right)} \leqslant c \varepsilon^{2}\|\psi\|_{\mathcal{C}_{\varepsilon}^{\ell, \alpha}\left(N_{0}\right)},
$$

and, for all $\ell^{\prime} \geqslant \ell$

$$
\left\|\psi_{\star}\right\|_{\mathcal{C}_{\varepsilon}^{\ell^{\prime}, \alpha}\left(N_{0}\right)} \leqslant c\|\psi\|_{\mathcal{C}_{\varepsilon}^{\ell, \alpha}\left(N_{0}\right)},
$$

where the constant $c$ depends on $\ell^{\prime}$ and $\ell$ but does not depend on $\Lambda^{\prime}$.

Indeed, using the first property of $R_{\theta}$ in (11.12), we get $\left\|\psi_{\star}\right\|_{\mathcal{C}^{2}\left(N_{0}\right)} \leqslant$ $c\|\psi\|_{\mathcal{C}^{2}\left(N_{0}\right)}$ and, for all $2 \leqslant k \leqslant \ell$, we have

$$
\left\|\nabla^{k} \psi_{\star}\right\|_{L^{\infty}\left(N_{0}\right)} \leqslant c\|\psi\|_{\mathcal{C}^{k, 0}\left(N_{0}\right)} \leqslant c \varepsilon^{2-k}\|\psi\|_{\mathcal{C}_{\varepsilon}^{\ell, \alpha}\left(N_{0}\right)},
$$


Now, for all $\ell \leqslant k \leqslant \ell+\alpha$, we use the second property of $R_{\theta}$ in (11.12) to get

$$
\left\|\nabla^{k} \psi_{\star}\right\|_{L^{\infty}\left(N_{0}\right)} \leqslant c \varepsilon^{\ell+\alpha-k}\|\psi\|_{\mathcal{C}^{\ell, \alpha}\left(N_{0}\right)} \leqslant c \varepsilon^{2-k}\|\psi\|_{\mathcal{C}_{\varepsilon}^{\ell, \alpha}\left(N_{0}\right)},
$$

which implies the second estimate (11.13). Finally, for all $\ell^{\prime} \leqslant \ell$, we use the third property of $R_{\theta}$ in (11.12) to show that

$$
\left\|\nabla^{k}\left(\psi_{\star}-\psi\right)\right\|_{L^{\infty}\left(N_{0}\right)} \leqslant c \varepsilon^{\ell+\alpha-k}\|\psi\|_{\mathcal{C}^{\ell, \alpha}\left(N_{0}\right)} \leqslant c \varepsilon^{2-k}\|\psi\|_{\mathcal{C}_{\varepsilon}^{\ell, \alpha}\left(N_{0}\right)},
$$

which proves (11.14). The proof of the claim is therefore complete.

In the case where $N$ has a nonempty boundary, this construction can be modified so that we can assume that $N_{\star}$ is an admissible hypersurface. Thanks to (11.13) and (11.14), we can apply all the above results to $N_{\star}$, provided $\Lambda^{\prime \prime}$ is chosen small enough. This yields the:

Proposition 11.8. Assume that $N$ satisfies $\left(H\left(2, \Lambda^{\prime \prime}\right)\right)$ and let $N_{\star}=R_{1 / \varepsilon} N$. Then, there exists $\varepsilon_{0}>0$ such that, for all $\varepsilon \in\left(0, \varepsilon_{0}\right)$, there exists an operator

$$
\mathbb{G}_{\varepsilon, N}: \mathcal{E}_{\varepsilon, N_{\star}}^{0, \alpha}(M) \longrightarrow\left[\mathcal{E}_{\varepsilon, N_{\star}}^{2, \alpha}(M)\right]_{0},
$$

satisfying

$$
\Pi_{\varepsilon, N_{\star}} \circ \mathbb{L}_{\varepsilon, N} \circ \mathbb{G}_{\varepsilon, N}=I .
$$

Furthermore,

$$
\left\|\mathbb{G}_{\varepsilon, N} w\right\|_{\mathcal{C}_{\varepsilon}^{2, \alpha}(M)} \leqslant c\|w\|_{\mathcal{C}_{\varepsilon}^{0, \alpha}(M)}
$$

and

$$
\left|S_{\varepsilon, N_{\star}} \circ \mathbb{L}_{\varepsilon, N} \circ \mathbb{G}_{\varepsilon, N} w\right|_{\mathcal{C}_{\varepsilon}^{0, \alpha}\left(N_{0}\right)} \leqslant c \varepsilon^{2}\|w\|_{\mathcal{C}_{\varepsilon}^{0, \alpha}(M)},
$$

for some constant $c>0$ which depends on $\Lambda^{\prime \prime}$ but does not depend on $\varepsilon$ nor on $N$.

Proof. We claim that

$$
\left\|\left(\mathbb{L}_{\varepsilon, N_{\star}}-\mathbb{L}_{\varepsilon, N}\right) w\right\|_{\mathcal{C}_{\varepsilon}^{0, \alpha}(M)} \leqslant c \varepsilon\|w\|_{\mathcal{C}_{\varepsilon}^{0, \alpha}(M)} .
$$

This follows from the fact that the difference of these two operators is equal to the difference of their potentials which involve functions depending on the distance to $N$ and $N_{\star}$. The result is then a consequence of the fact that difference $\psi_{\star}-\psi$ satisfies the estimate (11.13). 
Now, we apply the results of Proposition 11.6 to get for all $f \in$ $\mathcal{E}_{\varepsilon, N_{\star}}^{0, \alpha}(M)$ a solution $w \in\left[\mathcal{E}_{\varepsilon, N_{\star}}^{2, \alpha}(M)\right]_{0}$ of

$$
\Pi_{\varepsilon, N_{\star}} \circ \mathbb{L}_{\varepsilon, N_{\star}} w=f,
$$

with

$$
\|w\|_{\mathcal{C}_{\varepsilon}^{2, \alpha}(M)} \leqslant c\|f\|_{\mathcal{C}_{\varepsilon}^{0, \alpha}(M)}
$$

The existence of $\mathbb{G}_{\varepsilon, N}$ then follows from (11.16) together with a simple perturbation argument. It remains to check (11.15). But this follows at once from (11.16) together with the result of Proposition 11.7. q.e.d.

Observe that the mapping $\mathbb{G}_{\varepsilon, N}$ depends continuously on $N$. This essentially follows from the fact that, each step of the construction of this operator depends continuously on $N$.

\section{Moving the nodal set}

Assume that we are given an admissible hypersurface $N_{0}$. We define for convenience

$$
\mathbb{Q}_{\varepsilon}(N):=-\varepsilon^{2} \Delta u_{\varepsilon, N}+\frac{1}{2} W^{\prime}\left(u_{\varepsilon, N}\right),
$$

where $N$ is an admissible hypersurface which can be written as a normal graph over $N_{0}$. If $N_{\star}$ is the regularized hypersurface defined at the end of the previous section, we define, as in (11.10), the operator $S_{\varepsilon, N_{\star}}$ by

$$
S_{\varepsilon, N_{\star}}(u)(y):=\int_{\mathbb{R}} \chi(t) u(t, y) w_{\star}(t / \varepsilon) d t,
$$

where $(t, y)$ are (twisted) Fermi coordinates relative to $N_{\star}$ and $u$ is a function defined in $V_{\tau_{0}}(N)$. Since $N_{\star}$ is assumed to be a normal graph over $N_{0}$, any function on $N_{\star}$ can be identified with a function on $N_{0}$ and its norm can be evaluated using the norms defined in (10.2) or (10.3).

In this section we exploit the notion of nondegeneracy which have been defined in Definition 3.2 and Definition 3.3. There are two results which correspond to the two different notions of nondegeneracy. To start

with let us assume that we are dealing with an admissible nondegenerate minimal hypersurface $N_{0}$. We have: 
Lemma 12.1. Assume that $N_{0}$ is an admissible nondegenerate minimal hypersurface and that $\bar{c}>0$ is fixed. Then, there exists $\varepsilon_{0} \in(0,1)$ such that, for all $f \in \mathcal{C}^{0, \alpha}\left(N_{0}\right)$ satisfying

$$
|f|_{\mathcal{C}_{\varepsilon}^{0, \alpha}\left(N_{0}\right)} \leqslant \bar{c} \varepsilon^{2},
$$

one can find an admissible hypersurface $N$ satisfying

$$
S_{\varepsilon, N_{\star}} \mathbb{Q}_{\varepsilon}(N)=\varepsilon^{2} f
$$

where $N_{\star}=R_{1 / \varepsilon} N$. Moreover, if $\psi_{N}$ is the function whose graph is $N$, we have

$$
\left\|\psi_{N}\right\|_{\mathcal{C}_{\varepsilon}^{2, \alpha}\left(N_{0}\right)} \leqslant c \varepsilon^{-\alpha}\left(\varepsilon^{2}+|f|_{\mathcal{C}_{\varepsilon}^{0, \alpha}\left(N_{0}\right)}\right),
$$

for some constant $c>0$ which does not depend on $f$ nor on $\varepsilon$.

Proof. The proof of this result follows from the implicit function theorem. Granted the definition of $S_{\varepsilon, N_{\star}}$, we have to find and admissible hypersurface $N$ such that

$$
\int_{\mathbb{R}} \chi(t)\left(-\varepsilon^{2} \Delta u_{\varepsilon, N}+\frac{1}{2} W^{\prime}\left(u_{\varepsilon, N}\right)\right) w_{\star}(t / \varepsilon) d t=\varepsilon^{2} f,
$$

where $(t, y)$ are Fermi coordinates relative to $N_{\star}$. Using (10.5) we compute

$$
-\varepsilon^{2} \Delta u_{\varepsilon, N}+\frac{1}{2} W^{\prime}\left(u_{\varepsilon, N}\right)=\varepsilon n H_{N_{s(t, y)}} w_{\star}(t / \varepsilon)
$$

where $s(t, y)$ denotes the distance to of the point $(t, y)$ to $N$ and $N_{s}$ is the hypersurface parallel to $N$ at distance $s$. We now write $N$ as a graph over $N_{0}$ for some function $\psi$ and hence $N_{\star}$ is the normal graph over $N_{0}$ for the function $\psi_{\star}=R_{1 / \varepsilon} \psi$. We define

$$
A_{\varepsilon}(\psi):=n \int_{\mathbb{R}} \chi(\varepsilon t) H_{N_{s(\varepsilon t, y)}} w_{\star}^{2}(t) d t .
$$

so that the equation we have to solve reads

$$
A_{\varepsilon}(\psi)=f
$$

We claim that

$$
A_{\varepsilon}(\psi)=c_{\star} \mathcal{L}_{N_{0}} \psi+\mathcal{Q}(\varepsilon, \psi)
$$


where $\mathcal{L}_{N_{0}}$ is the Jacobi operator about $N_{0}$, where

$$
c_{\star}=\int_{\mathbb{R}} w_{\star}^{2} d t
$$

and where $\mathcal{Q}$ satisfies

$$
|\mathcal{Q}(\varepsilon, 0)|_{\mathcal{C}_{\varepsilon}^{0, \alpha}\left(N_{0}\right)} \leqslant c \varepsilon^{2}
$$

and

$$
\begin{aligned}
& \left|\mathcal{Q}\left(\varepsilon, \psi^{\prime}\right)-\mathcal{Q}(\varepsilon, \psi)\right|_{\mathcal{C}_{\varepsilon}^{0, \alpha}\left(N_{0}\right)} \\
& \leqslant c\left(\varepsilon^{2}+\|\psi\|_{\mathcal{C}_{\varepsilon}^{2, \alpha}\left(N_{0}\right)}+\left\|\psi^{\prime}\right\|_{\mathcal{C}_{\varepsilon}^{2, \alpha}\left(N_{0}\right)}\right)\left\|\psi^{\prime}-\psi\right\|_{\mathcal{C}_{\varepsilon}^{2, \alpha}\left(N_{0}\right)},
\end{aligned}
$$

for some constant $c>0$ which depends on $\bar{c}$ but does not depend on $\varepsilon$ nor on $\psi, \psi^{\prime}$ satisfying

$$
\|\psi\|_{\mathcal{C}_{\varepsilon}^{2, \alpha}\left(N_{0}\right)}+\left\|\psi^{\prime}\right\|_{\mathcal{C}_{\varepsilon}^{2, \alpha}\left(N_{0}\right)} \leqslant 1
$$

To obtain this expansion, we first use the second estimate in (11.13) to reduce to the case where $N_{\star}$ is replaced by $N$, i.e., $A_{\varepsilon}(\psi)$ is replaced by

$$
\widetilde{A}_{\varepsilon}(\psi):=n \int_{\mathbb{R}} \chi(\varepsilon s) H_{N_{\varepsilon s}} w_{\star}^{2}(s) d s .
$$

If $D_{\varepsilon}(\psi):=\widetilde{A}_{\varepsilon}(\psi)-A_{\varepsilon}(\psi)$, we have $D_{\varepsilon}(0)=0$ and it follows from (11.13) that

$$
\left|D_{\varepsilon}\left(\psi^{\prime}\right)-D_{\varepsilon}(\psi)\right|_{\mathcal{C}_{\varepsilon}^{0, \alpha}\left(N_{0}\right)} \leqslant c \varepsilon^{2}\left\|\psi^{\prime}-\psi\right\|_{\mathcal{C}_{\varepsilon}^{2, \alpha}\left(N_{0}\right)},
$$

We now use the expansion

$$
H_{N_{t}}=H_{N}+t Q_{1}(\psi ; y)+t^{2} Q_{2}(\psi ; t, y),
$$

where $Q_{1}(\psi ; y)$ does not depend on $t$. In particular

$$
n \int_{\mathbb{R}} \chi(\varepsilon s) \varepsilon s Q_{1}(\psi ; y) w_{\star}^{2}(s) d s \equiv 0,
$$

and, if we define

$$
E_{\varepsilon}(\psi):=n \varepsilon^{2} \int_{\mathbb{R}} \chi(\varepsilon s) s^{2} Q_{2}(\psi ; \varepsilon s, y) w_{\star}^{2}(s) d s,
$$


we obtain $\left|E_{\varepsilon}(0)\right|_{\mathcal{C}_{\varepsilon}^{0, \alpha}\left(N_{0}\right)} \leqslant c$ and the estimate

$$
\left|E_{\varepsilon}\left(\psi^{\prime}\right)-E_{\varepsilon}(\psi)\right|_{\mathcal{C}_{\varepsilon}^{0, \alpha}\left(N_{0}\right)} \leqslant c \varepsilon^{2}\left\|\psi^{\prime}-\psi\right\|_{\mathcal{C}_{\varepsilon}^{2, \alpha}\left(N_{0}\right)},
$$

Finally, we use the fact that

$$
n H_{N}=n H_{N_{0}}+\mathcal{L}_{N_{0}} \psi+Q(\psi) \text {, }
$$

and we check that

$$
F_{\varepsilon}(\psi):=n Q(\psi) \int_{\mathbb{R}} \chi(\varepsilon s) w_{\star}^{2}(s) d s,
$$

satisfies $Q(0)=0$ and

$$
\begin{aligned}
& \left|F_{\varepsilon}\left(\psi^{\prime}\right)-F_{\varepsilon}(\psi)\right|_{\mathcal{C}_{\varepsilon}^{0, \alpha}\left(N_{0}\right)} \\
& \quad \leqslant c\left(\|\psi\|_{\mathcal{C}_{\varepsilon}^{2, \alpha}\left(N_{0}\right)}+\left\|\psi^{\prime}\right\|_{\mathcal{C}_{\varepsilon}^{2, \alpha}\left(N_{0}\right)}\right)\left\|\psi^{\prime}-\psi\right\|_{\mathcal{C}_{\varepsilon}^{2, \alpha}\left(N_{0}\right)},
\end{aligned}
$$

We have assumed that the operator

$$
\mathcal{L}_{N_{0}}:\left[\mathcal{C}^{2, \alpha}\left(N_{0}\right)\right]_{0} \longrightarrow \mathcal{C}^{0, \alpha}\left(N_{0}\right)
$$

is an isomorphism. Here the subscript 0 means that we are considering functions which satisfy $\mathcal{B}_{N_{0}}(\psi)=0$ on $\partial N_{0}$, if this boundary is not empty. This also implies that

$$
\mathcal{L}_{N_{0}}:\left[\mathcal{C}_{\varepsilon}^{2, \alpha}\left(N_{0}\right)\right]_{0} \longrightarrow \mathcal{C}_{\varepsilon}^{0, \alpha}\left(N_{0}\right),
$$

is an isomorphism, the norm of whose inverse is bounded by a constant times $\varepsilon^{-\alpha}$ when the space $\mathcal{C}_{\varepsilon}^{2, \alpha}\left(N_{0}\right)$ is endowed with the norm $\|\cdot\|_{\mathcal{C}_{\varepsilon}^{2, \alpha}\left(N_{0}\right)}$ defined in (10.2) and the space $\mathcal{C}_{\varepsilon}^{0, \alpha}\left(N_{0}\right)$ is endowed with the norm $|\cdot|_{\mathcal{C}_{\varepsilon}^{0, \alpha}\left(N_{0}\right)}$ defined in (10.3). Indeed, if $\mathcal{L}_{N_{0}} v=g$ and if $|g|_{\mathcal{C}_{\varepsilon}^{0, \alpha}\left(N_{0}\right)} \leqslant 1$, then $\|g\|_{\mathcal{C}^{0, \alpha}\left(N_{0}\right)} \leqslant \varepsilon^{-\alpha}$ and hence $\|v\|_{\mathcal{C}^{2, \alpha}\left(N_{0}\right)}$ is bounded by a constant times $\varepsilon^{-\alpha}$. In particular, $\|v\|_{\mathcal{C}_{\varepsilon}^{2, \alpha}\left(N_{0}\right)}$ is also bounded by a constant times $\varepsilon^{-\alpha}$.

The proof of the result is now a corollary of the fixed point theorem for contraction mappings, provided $\varepsilon$ is chosen small enough. q.e.d.

We now state the corresponding result for volume-nondegenerate constant mean curvature hypersurfaces. 
Lemma 12.2. Assume that $N_{0}$ is an admissible constant mean curvature hypersurface which is volume-nondegenerate. We define

$$
\lambda_{\star}:=\frac{1}{2} c_{\star} n H_{N_{0}}
$$

where

$$
c_{\star}:=\int_{-1}^{1} \sqrt{W(s)} d s
$$

Then, there exists $\varepsilon_{0} \in(0,1)$ and $\bar{c}>0$ such that for all $f \in \mathcal{C}^{0, \alpha}\left(N_{0}\right)$ and all $\mu \in \mathbb{R}$, satisfying

$$
|f|_{\mathcal{C}_{\varepsilon}^{0, \alpha}\left(N_{0}\right)}+|\mu| \leqslant \bar{c} \varepsilon
$$

one can find an admissible hypersurface $N$ and a constant $\lambda$ such that

$$
S_{\varepsilon, N_{\star}}\left(\mathbb{Q}_{\varepsilon}(N)-\varepsilon \lambda\right)=\varepsilon^{2} f,
$$

and

$$
\int_{M} u_{\varepsilon, N} d v_{g}=c_{0}|M|+\mu .
$$

Moreover, if $\psi_{N}$ is the function whose graph is $N$, we have

$$
\left\|\psi_{N}\right\|_{\mathcal{C}_{\varepsilon}^{2, \alpha}\left(N_{0}\right)} \leqslant c\left(|f|_{\mathcal{C}_{\varepsilon}^{0, \alpha}\left(N_{0}\right)}+|\mu|\right)
$$

for some constant $c>0$ which does not depend on the data $f, \mu$ nor on $\varepsilon$.

Proof. We now define

$$
A_{\varepsilon}(\psi):=n \int_{\mathbb{R}} \chi(\varepsilon t) H_{N_{s(\varepsilon t, y)}} w_{\star}^{2}(t) d t-\lambda \int_{\mathbb{R}} \chi(\varepsilon t) w_{\star}(t) d t,
$$

which can be expanded as

$$
A_{\varepsilon}(\psi)=n H_{N_{0}} \int_{\mathbb{R}} w_{\star}^{2}(t) d t-\varepsilon \lambda \int_{\mathbb{R}} w_{\star}(t) d t+c_{\star} \mathcal{L}_{N_{0}} \psi+\mathcal{Q}(\varepsilon, \psi),
$$

where $\mathcal{Q}(\varepsilon, \psi)$ satisfies (12.3) and (12.4). Now, observe that

$$
\int_{\mathbb{R}} w_{\star} d t=2
$$


and also that

$$
\int_{\mathbb{R}} w_{\star}^{2} d t=\int_{\mathbb{R}} \sqrt{W^{\prime}\left(u_{\star}\right)} \partial_{t} u_{\star} d t=\int_{-1}^{1} \sqrt{W^{\prime}(s)} d s=c_{\star} .
$$

Granted the definition of $\lambda_{\star}$, we can write

$$
A_{\varepsilon}(\psi)=c_{\star} \mathcal{L}_{N_{0}} \psi-2\left(\lambda-\lambda_{\star}\right)+\mathcal{Q}(\varepsilon, \psi) .
$$

We now expand

$$
\int_{M} u_{\varepsilon, N} d v_{g}=c_{0}|M|-2 \int_{N_{0}} \psi d a_{g}+O(\varepsilon, \psi)
$$

where the operator $O$ satisfies

$$
|O(\varepsilon, 0)| \leqslant c \varepsilon^{2}, \quad \text { and } \quad\left|O\left(\varepsilon, \psi^{\prime}\right)-O(\varepsilon, \psi)\right| \leqslant c \varepsilon^{2}\left\|\psi^{\prime}-\psi\right\|_{\mathcal{C}_{\varepsilon}^{2, \alpha}\left(N_{0}\right)},
$$

for some constant $c>0$ which depends on $\bar{c}$ but does not depend on $\varepsilon$ nor on $\psi, \psi^{\prime}$ satisfying

$$
\|\psi\|_{\mathcal{C}_{\varepsilon}^{2, \alpha}\left(N_{0}\right)}+\left\|\psi^{\prime}\right\|_{\mathcal{C}_{\varepsilon}^{2, \alpha}\left(N_{0}\right)} \leqslant 1
$$

The proof now follows exactly the proof of the previous Lemma when volume-nondegeneracy replaces nondegeneracy. We omit the details.

q.e.d.

\section{The nonlinear problem}

\subsection{The proof of Theorem 4.1}

Assume that we are given a volume-nondegenerate admissible minimal hypersurface $N_{0}$ in $M$. We would like to solve the nonlinear problem

$$
-\varepsilon^{2} \Delta_{g}\left(u_{\varepsilon, N}+v\right)+\frac{1}{2} W^{\prime}\left(u_{\varepsilon, N}+v\right)=0
$$

in $M$. In addition, we want $u_{\varepsilon, N}+v$ to have 0 Neumann boundary data on $\partial M$ if this later is not empty. This means that, for all $\varepsilon$ small enough, we would like to find an admissible hypersurface $N$ close to $N_{0}$ and a function $v$ close to 0 satisfying (13.1). 
Recall that we have defined the nonlinear operator

$$
\mathbb{Q}_{\varepsilon}(N):=-\varepsilon^{2} \Delta_{g} u_{\varepsilon, N}+\frac{1}{2} W^{\prime}\left(u_{\varepsilon, N}\right),
$$

which corresponds to the error we produce when we consider $u_{\varepsilon, N}$ as a solution of (13.1). We define

$$
\widetilde{\mathbb{Q}}_{\varepsilon}(N, v):=\frac{1}{2}\left(W^{\prime}\left(u_{\varepsilon, N}+v\right)-W^{\prime}\left(u_{\varepsilon, N}\right)-W^{\prime \prime}\left(u_{\varepsilon, N}\right) v\right),
$$

which is nothing but the Taylor expansion of the nonlinearity $W^{\prime}$ at $u_{\varepsilon, N}$.

These definitions being understood, the equation we have to solve reads

$$
\mathbb{Q}_{\varepsilon}(N)+\mathbb{L}_{\varepsilon, N} v+\widetilde{\mathbb{Q}}_{\varepsilon}(N, v)=0 .
$$

Using the projection $\Pi_{\varepsilon, N_{\star}}$ defined in (11.2) and the operator $S_{\varepsilon, N_{\star}}$ defined in (12.2), we conclude that (13.1) is equivalent to the system

$$
\left\{\begin{aligned}
\Pi_{\varepsilon, N_{\star}} \circ \mathbb{L}_{\varepsilon, N} v & =-\Pi_{\varepsilon, N_{\star}}\left(\mathbb{Q}_{\varepsilon}(N)+\widetilde{\mathbb{Q}}_{\varepsilon}(N, v)\right) \\
S_{\varepsilon, N_{\star}} \mathbb{Q}_{\varepsilon}(N) & =-S_{\varepsilon, N_{\star}}\left(\mathbb{L}_{\varepsilon, N} v+\widetilde{\mathbb{Q}}_{\varepsilon}(N, v)\right),
\end{aligned}\right.
$$

where $N_{\star}=R_{1 / \varepsilon} N$ is the hypersurface obtained at the end of $\S 11$ from $N$.

This system will be solved using a fixed point argument. The key result which allows one to apply a fixed point theorem is the following estimate:

Lemma 13.1. There exists a constant $c_{0}>0$ such that, for all $\varepsilon \in(0,1)$

$$
\left\|\mathbb{Q}_{\varepsilon}\left(N_{0}\right)\right\|_{\mathcal{C}_{\varepsilon}^{0, \alpha}(M)} \leqslant \frac{c_{0}}{2} \varepsilon^{2},
$$

and, given $c_{2}>0$, there exists $\varepsilon_{0}>0$ such that, for all $\varepsilon \in\left(0, \varepsilon_{0}\right)$ and all admissible hypersurface $N$ satisfying

$$
\|N\|_{\mathcal{C}_{\varepsilon}^{2, \alpha}\left(N_{0}\right)} \leqslant c_{2} \varepsilon^{2-\alpha},
$$

we have

$$
\left\|\mathbb{Q}_{\varepsilon}(N)\right\|_{\mathcal{C}_{\varepsilon}^{0, \alpha}(M)} \leqslant c_{0} \varepsilon^{2}
$$


Proof. It follows from (10.5) that

$$
\mathbb{Q}_{\varepsilon}(N):=-\varepsilon^{2} \Delta_{g} u_{\varepsilon, N}+\frac{1}{2} W^{\prime}\left(u_{\varepsilon, N}\right)=n \varepsilon H_{N_{t}} w_{\star}(t / \varepsilon),
$$

in $V_{\tau_{0} / 2}(N)$, where $N_{t}$ is the hypersurface parallel to $N$ at distance t. Moreover $\mathbb{Q}_{\varepsilon}(N) \equiv 0$ in $M-V_{\tau_{0}}(N)$. Since $N_{0}$ is minimal and $\|N\|_{\mathcal{C}_{\varepsilon}^{2, \alpha}\left(N_{0}\right)} \leqslant c_{2} \varepsilon^{2-\alpha}$, we estimate

$$
\left|H_{N_{t}}\right| \leqslant c\left(|t|+c_{2} \varepsilon^{2-\alpha}\right)
$$

$V_{\tau_{0}}(N)-V_{\tau_{0} / 2}(N)$ and this already implies that

$$
\left\|H_{N_{t}} w_{\star}(t / \varepsilon)\right\|_{L^{\infty}(M)} \leqslant c\left(\varepsilon+c_{2} \varepsilon^{2-\alpha}\right) .
$$

(The estimate in $V_{\tau_{0}}(N)-V_{\tau_{0} / 2}(N)$ is easy to get since $\mathbb{Q}_{\varepsilon}(N)$ is exponentially small in this set). The estimates for the Hölder derivative follows similarly.

It now suffices to apply this estimate to $N_{0}$ itself to obtain (13.5), while (13.6) follows at once by taking $\varepsilon$ to be small enough. $\quad$ q.e.d.

Assume that we are given $v \in \mathcal{C}_{\varepsilon}^{0, \alpha}(M)$ and $N$ an admissible hypersurface close to $N_{0}$. To make things quantitatively precise, we assume that

$$
\|v\|_{\mathcal{C}_{\varepsilon}^{0, \alpha}(M)} \leqslant c_{1} \varepsilon^{2}, \quad \text { and } \quad\|N\|_{\mathcal{C}_{\varepsilon}^{2, \alpha}(M)} \leqslant c_{2} \varepsilon^{2-\alpha} .
$$

For some constant $c_{1}$ and $c_{2}$ which will be fixed shortly. We apply the result of Proposition 11.8 and Lemma 12.1 to find $\widetilde{v}$ and $\widetilde{N}$ solutions of

$$
\left\{\begin{aligned}
\Pi_{\varepsilon, N_{\star}} \circ \mathbb{L}_{\varepsilon, N} \widetilde{v} & =-\Pi_{\varepsilon, N_{\star}}\left(\mathbb{Q}_{\varepsilon}(N)+\widetilde{\mathbb{Q}}_{\varepsilon}(N, v)\right) \\
S_{\varepsilon, \widetilde{N}_{\star}} \mathbb{Q}_{\varepsilon}(\tilde{N}) & =-S_{\varepsilon, N_{\star}}\left(\mathbb{L}_{\varepsilon, N} v+\widetilde{\mathbb{Q}}_{\varepsilon}(N, v)\right) .
\end{aligned}\right.
$$

Using the fact that $\widetilde{Q}_{\varepsilon}$ is quadratic in $v$ together with Lemma 13.1, we easily get the estimates

$$
\begin{aligned}
& \|\widetilde{v}\|_{\mathcal{C}_{\varepsilon}^{2, \alpha}(M)} \leqslant c\left(c_{0} \varepsilon^{2}+C_{c_{1}} \varepsilon^{4}\right), \quad \text { and } \\
& \|\widetilde{N}\|_{\mathcal{C}_{\varepsilon}^{2, \alpha}(M)} \leqslant c \varepsilon^{-\alpha}\left(c_{1} \varepsilon^{2}+C_{c_{1}} \varepsilon^{3}\right) .
\end{aligned}
$$

provided $\varepsilon$ is chosen small enough. Here the constant $c>0$ neither depends on $c_{1}$ nor on $c_{2}$ and the constant $C_{c_{1}}$ depends on $c_{1}$. 
It follows at once that, if

$$
c_{1}=2 c c_{0} \quad \text { and } \quad c_{2}=2 c c_{1},
$$

and if $\varepsilon$ is chosen small enough, this produces a continuous mapping from the set of $(v, N) \in \mathcal{C}_{\varepsilon}^{2, \alpha}(M) \times \mathcal{C}_{\varepsilon}^{2, \alpha}\left(N_{0}\right)$ satisfying (13.7) into itself. If this mapping were compact, we would obtain a fixed point through Schauder's fixed point theorem. However, in our case the mapping just fails to be compact since no regularity is gained through the iteration process. To overcome this problem, we use once more the smoothing operators $R_{\theta}$ which have been introduced in the proof of Proposition 11.8. We define instead $\widetilde{v}$ and $\widetilde{N}$ to be the solutions of

$$
\left\{\begin{aligned}
\Pi_{\varepsilon, N_{\star}} \circ \mathbb{L}_{\varepsilon, N} \widetilde{v} & =-\Pi_{\varepsilon, N_{\star}} \circ R_{\theta}\left(\mathbb{Q}_{\varepsilon}(N)+\widetilde{\mathbb{Q}}_{\varepsilon}(N, v)\right) \\
S_{\varepsilon, \widetilde{N}_{\star}} \mathbb{Q}_{\varepsilon}(\widetilde{N}) & =-R_{\theta} \circ S_{\varepsilon, N_{\star}}\left(\mathbb{L}_{\varepsilon, N} v+\widetilde{\mathbb{Q}}_{\varepsilon}(N, v)\right) .
\end{aligned}\right.
$$

We fix $\alpha^{\prime}>\alpha$ and choose $\theta$ so that $C \theta^{\alpha^{\prime}-\alpha}=2$, where $C$ is the constant which appears in the estimate of the smoothing operator $R_{\theta}$. If $c_{1}=4 c c_{0}$ and $c_{2}=4 c c_{1}$, this produces, for all $\varepsilon$ small enough, a continuous mapping from the set of $(v, N) \in \mathcal{C}_{\varepsilon}^{2, \alpha}(M) \times \mathcal{C}_{\varepsilon}^{2, \alpha}\left(N_{0}\right)$ satisfying (13.7) into itself, but this time the mapping is compact. We conclude that there exists a fixed point $\left(v_{\theta}, N_{\theta}\right)$. Finally, we pass to the limit as $\theta$ tends to $+\infty$ (i.e., as $\alpha^{\prime}$ tends to $\alpha$ ). The solutions $\left(v_{\theta}, N_{\theta}\right)$ of (13.9) being uniformly bounded in $\mathcal{C}_{\varepsilon}^{2, \alpha}(M) \times \mathcal{C}_{\varepsilon}^{2, \alpha}\left(N_{0}\right)$, we can extract a subsequence which converges to $(v, N)$ in $\mathcal{C}_{\varepsilon}^{2, \beta}(M) \times \mathcal{C}_{\varepsilon}^{2, \beta}\left(N_{0}\right)$, for some fixed $\beta<\alpha$. The limit $(v, N)$ is then a solution of our problem.

\subsection{The proof of Theorem 4.2}

Assume that we are given a volume-nondegenerate admissible constant mean curvature hypersurface $N_{0}$ in $M$. We would like to solve the nonlinear problem

$$
-\varepsilon^{2} \Delta\left(u_{\varepsilon, N}+v\right)+\frac{1}{2} W^{\prime}\left(u_{\varepsilon, N}+v\right)=\varepsilon \lambda,
$$

in $M$, with $u_{\varepsilon, N}+v$ having 0 Neumann boundary data if $\partial M$ is not empty. This equation has to be complimented with the constraint

$$
\int_{M}\left(u_{\varepsilon, N}+v\right) d v_{g}=c_{0}|M|
$$


where the constant $c_{0} \in(-1,1)$ is fixed so that

$$
c_{0}|M|=\left|M^{+}\left(N_{0}\right)\right|-\left|M^{-}\left(N_{0}\right)\right| .
$$

Again, in order to solve (13.10), we write the equation as a fixed point problem

$$
\left\{\begin{aligned}
\Pi_{\varepsilon, N_{\star}} \circ \mathbb{L}_{\varepsilon, N} v & =-\Pi_{\varepsilon, N_{\star}}\left(\mathbb{Q}_{\varepsilon}(N)-\varepsilon \lambda+\mathbb{Q}_{\varepsilon}(N, v)\right) \\
S_{\varepsilon, N_{\star}}\left(\mathbb{Q}_{\varepsilon}(N)-\varepsilon \lambda\right) & =-S_{\varepsilon, N_{\star}}\left(\mathbb{L}_{\varepsilon, N} v+\widetilde{\mathbb{Q}}_{\varepsilon}(N, v)\right) \\
\int_{M} u_{\varepsilon, N} d v_{g} & =c_{0}|M|-\int_{M} v d v_{g} .
\end{aligned}\right.
$$

The proof of the existence of a fixed point is identical to the proof of the previous result, with Lemma 12.1 replaced by Lemma 12.2. The only difference being that the hypersurface $N_{0}$ does not have 0 mean curvature anymore and this implies that Lemma 13.1 has to be replaced by

Lemma 13.2. There exists a constant $c_{0}>0$ such that, for all $\varepsilon \in(0,1)$

$$
\left\|\mathbb{Q}_{\varepsilon}\left(N_{0}\right)\right\|_{\mathcal{C}_{\varepsilon}^{0, \alpha}(M)} \leqslant \frac{c_{0}}{2} \varepsilon
$$

and, given $c_{2}>0$, there exists $\varepsilon_{0}>0$ such that, for all $\varepsilon \in\left(0, \varepsilon_{0}\right)$ and all admissible hypersurface $N$ satisfying

$$
\|N\|_{\mathcal{C}_{\varepsilon}^{2, \alpha}\left(N_{0}\right)} \leqslant c_{2} \varepsilon^{1-\alpha}
$$

we have

$$
\left\|\mathbb{Q}_{\varepsilon}(N)\right\|_{\mathcal{C}_{\varepsilon}^{0, \alpha}(M)} \leqslant c_{0} \varepsilon
$$

The fact that we do not get an estimate as good as the one obtained in Lemma 13.1 is a consequence of the fact that the mean curvature of $N_{0}$ is not necessarily equal to 0 .

This implies that the condition (13.7) has to be replaced by

$$
\|v\|_{\mathcal{C}_{\varepsilon}^{0, \alpha}(M)} \leqslant c_{1} \varepsilon, \quad \text { and } \quad\|N\|_{\mathcal{C}_{\varepsilon}^{2, \alpha}(M)} \leqslant c_{2} \varepsilon^{1-\alpha} .
$$

Details are left to the reader. 


\section{References}

[1] S. Alinhac \& P. Gérard, Opérateurs pseudo-différentiels et théorème de NashMoser, InterEditions/Editions du CNRS, 1991, MR 93g:35001, Zbl 0791.47044.

[2] S. Allen \& J.W. Cahn, A microspopic theory for antiphase boundary motion and its application to antiphase domain coarsening, Acta. Metall. 27 (1979) 1084-1095.

[3] G. Anzellotti, S. Baldo \& G. Orlandi, Г-asymptotic developments, the CahnHilliard functional, and curvatures, J. Math. Anal. Appl. 197(3) (1996) 908-924, MR 96k:49064, Zbl 0857.49007.

[4] S. Baldo, Minimal interface criterion for phase transitions in mixtures of CahnHilliard fluids, Ann. Inst. H. Poincaré Anal. Non Linéaire 7(2) (1990) 67-90, MR 91j:76005, Zbl 0702.49009.

[5] J.L. Barbosa, M.P. do Carmo \& Jost Eschenburg, Stability of hypersurfaces of constant mean curvature in Riemannian manifolds, Math. Z. 197(1) (1988) 123138, MR 88m:53109, Zbl 0653.53045.

[6] S. Brendle, On the construction of solutions to the Yang-Mills equations in higher dimensions, math.DG/0302093.

[7] S. Brendle, On solutions to the Ginzburg-Landau equations in higher dimensions, math.DG/0302070.

[8] J.W. Cahn \& J.E. Hilliard, Free energy of a nonuniform system I. Interfacial free energy, J. Chem. Phys. 28 (1958) 258-267.

[9] D. Gilbarg \& N.S. Trudinger, Elliptic partial differential equations of second order, second ed., Grundlehren der Mathematischen Wissenschaften [Fundamental Principles of Mathematical Sciences], Vol. 224, Springer-Verlag, Berlin, 1983, MR 86c:35035, Zbl 0562.35001.

[10] S.G. Krantz \& H.R. Parks, Distance to $\mathcal{C}^{k}$ hypersurfaces, J. Diff. Equations, 40(1) (1981) 116-120, MR 82h:58005, Zbl 0431.57009.

[11] M. Kowalczyk, On the existence and Morse index of solutions to the Allen-Cahn equation in two dimensions, to appear in Annali di Matematica Pura et Aplicata.

[12] S. Luckhaus \& L. Modica, The Gibbs-Thompson relation within the gradient theory of phase transitions, Arch. Rational Mech. Anal. 107(1) (1989) 71-83, MR 90k:49041, Zbl 0681.49012.

[13] S. Luckhaus \& L. Modica, The Gibbs-Thompson relation within the gradient theory of phase transitions, Arch. Rational Mech. Anal. 107(1) (1989) 71-83, MR 90k:49041, Zbl 0681.49012.

[14] A. Malchiodi \& M. Montenegro, Boundary concentration phenomena for a singularly perturbed elliptic problem, Comm. Pure and Applied Mathematics, 55(12) (2002) 1507-1568, MR 2003g:35005. 
[15] L. Modica, The gradient theory of phase transitions and the minimal interface criterion, Arch. Rational Mech. Anal. 98(2) (1987) 123-142, MR 88f:76038, Zbl 0616.76004 .

[16] L. Modica, Gradient theory of phase transitions with boundary contact energy, Ann. Inst. H. Poincaré Anal. Non Linéaire 4(5) (1987) 487-512, MR 89c:76108, Zbl 0642.49009.

[17] R. Mazzeo \& F. Pacard, A construction of singular solutions for a semilinear elliptic equation using asymptotic analysis, J. Differential Geom. 44(2) (1996) 331-370, MR 98a:35040, Zbl 0869.35040.

[18] A. Ros \& R. Souam, On stability of capillary surfaces in a ball, Pacific J. Math. 178(2) (1997) 345-361, MR 98c:58029, Zbl 0885.53010, Zbl 0930.53007.

[19] J.S. Rowlinson, Translation of J.D. van der Waals' "The thermodynamic theory of capillarity under the hypothesis of a continuous variation of density", J. Statist. Phys. 20(2) (1979) 197-244, MR 82e:01128.

[20] L. Schwartz, Théorie des distributions, Publications de l'Institut de Mathématique de l'Université de Strasbourg, IX-X, Hermann, Paris, 1966, MR 35 \#730, Zbl 1049.09501.

[21] P. Sternberg, The effect of a singular perturbation on nonconvex variational problems, Arch. Rational Mech. Anal. 101(3) (1988) 209-260, MR 89h:49007, Zbl 0647.49021.

[22] C.H. Taubes, Gr $\Longrightarrow$ SW: from pseudo-holomorphic curves to Seiberg-Witten solutions, J. Differential Geom. 51(2) (1999) 203-334, MR 2000i:53123.

[23] B. White, The space of minimal submanifolds for varying Riemannian metrics, Indiana Univ. Math. J. 40(1) (1991) 161-200, MR 92i:58028, Zbl 0742.58009.

Centre de Mathématiques

Faculté de Sciences et Technologie Université Paris XII - Val de Marne 94010 CReteil CEDEX

FRANCE

Departamento de Geometría y Topología Facultad de Ciencias Universidad DE GRANADA E-18071 GRANADA ESPAÑA 